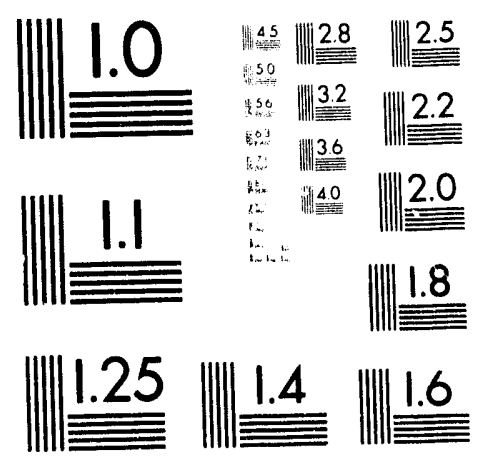



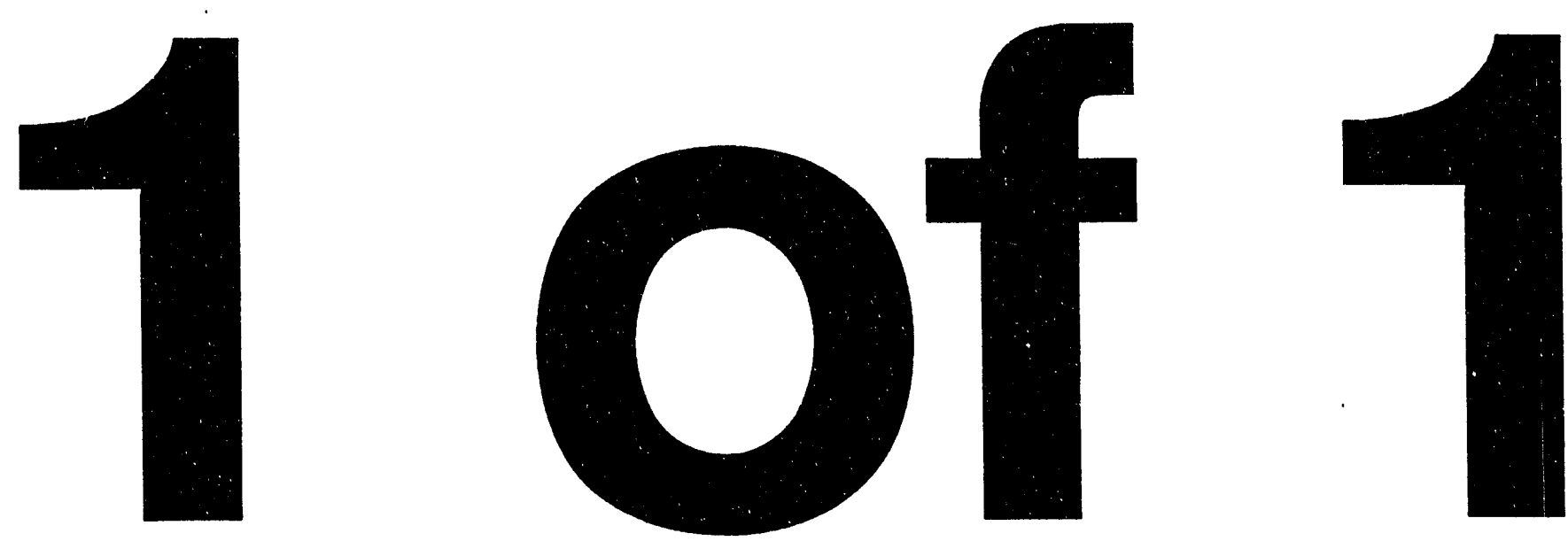


\title{
SEARCH FOR PSEUDOSCALAR COLD DARK MATTER
}

\author{
Karl van Bibber, LLNL \\ Wolfgang Stoeffi, LLNL
}

\section{LLNL Collaborators:}

P.L. Anthony, R.E. Patrick, D.S. Ravenscroft, S.S. Shen, D.S. Slack, S.W. Ferguson

\section{July 2, 1993}

This is an informal report intended primarily for internal or limited external distribution. The opinions and conclusions stated are those of the author and may or may not be those of the Laboratory.

Work performed under the auspices of the U.S. Department of Energy by the Lawrence Livermore National Laboratory under Contract W-7405-Eng-48. 


\section{DISCLAIMER}

This document was prepared as an account of work sponsored by an agency of the United States Government. Neither the United States Government nor the University of California nor any of their employees, makes any warranty, express or implied, or assumes any legal liability or responsibility for the accuracy, completeness, or usefulness of any information, apparatus, product, or process disclosed, or represents that its use would not infringe privately owned rights. Reference herein to any specific commercial products, process, or service by trade name, trademark, manufacturer, or otherwise, does not necessarily constitute or imply its endorsement, recommendation, or fa voring by the United States Government or the University of California. The views and opinions of authors expressed herein do not necessarily state or reflect those of the United States Government or the University of California, and shall not be used for advertising or product endorsement purposes.

This report has been reproduced

directly from the best available copy.

Available to DOE and DOE contractors from the Office of Scientific and Technical Information

P.O. Box 62, Oak Ridge, TN 37831

Prices a vailable from (615) 576-8401, FTS 626-8401

Available to the public from the

National Technical Information Service

U.S. Department of Commerce

5285 Port Royal Rd.,

Springfield, VA 22161 
Proposal (New) for Physics Exploratory Research in the Disciplines

\section{SEARCH FOR PSEUDOSCALAR COLD DARK MATTER}

Principal Investigators:

Karl van Bibber (E-Div.), Wolfgang Stoeffl (Nucl. Chem.)

LLNL Collaborators:

P.L. Anthony (E. Div.), R.E. Patrick, D.S. Ravenscroft, S.S. Shen, D.S. Slack, S.W. Ferguson (MFE Prog.)

Collaborating Institutions:

University of Florida

Institute for Nuclear Research of the Russian Academy of Sciences

Massachusetts Institute of Technology

University of Chicago

Fermi National Accelerator Laboratory

Lawrence Berkeley Laboratory

University of California Berkeley

Stanford Linear Accelerator Center

Wang NMR, Inc.

May 29, 1992

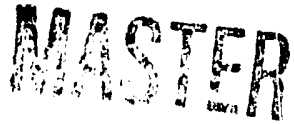




\title{
SEARCH FOR PSEUDOSCALAR COLD DARK MATTER
}

May 29, 1992

\begin{abstract}
All dynamical evidence points to the conclusion that the predominant form of matter in the universe is in a non-luminous form. Furthermore, large scale deviations from uniform Hubble flow, and the recent COBE reports of inhomogeneities in the cosmic microwave background strongly suggest that we live in an exactly closed universe. If this is true, then ordinary baryonic matter could only be a minority component (10\% at most) of the missing mass, and that what constitutes the majority of the 'dark matter' must involve new physics, e.g. a previously undiscovered elementary particle, massive neutrinos, etc. The axion, a light pseudoscalar particle, is one of very few wellmotivated candidates which may comprise the dark matter. Additionally it is a 'cold' dark-matter candidate, i.e. non-relativistic from the time of the early universe, which is preferred by the COBE data. We propose to construct and operate an experiment to search for axions which may constitute the dark matter of our own galaxy. This would be the first axion search with cosmologically significant sensitivity, and at present we have no competition in the world. As proposed by Sikivie (1983), dark-matter axions may be detected by their stimulated conversion into monochromatic microwave photons in a tunable high- $Q$ cavity inside a strong magnetic field. Our ability to mount an experiment quickly and take data within one year is due to a confluence of three factors. The first is the availability of a compact high field superconducting magnet (the NASA SUMMA coils), and a local industrial partner, Wang NMR, who can make a very thermally efficient and economical cryostat for it. The second is an ongoing joint venture with the Institute for Nuclear Research of the Russian Academy of Sciences to do $R \& D$ on metalized precision-formed ceramic microwave cavities for the axion search, and INR has commited to providing all the microwave cavity arrays for this experiment, should this proposal be approved. The third is a commitment of very substantial startup capital monies from MIT for all of the state-of-the-art ultra-low noise microwave electronics, to one of our outstanding young collaborators who is joining their faculty. The first year's activities, for which our budget request is $\$ 300 \mathrm{~K}$ operating monies and $\$ 50 \mathrm{~K}$ capital, consists of construction and testing of all three basic components of the detector. The actual experimental search begins at the outset of the second year, with each of the five cavity arrays requiring approximately six months of operation to cover the proposed mass range.
\end{abstract}




\section{Introduction}

It is now virtually certain that at least $90 \%$ and perhaps even $99 \%$ of the mass of the universe is in a non-luminous form. The evidence for this 'dark matter' is based on verv fundamental dynamical grounds, i.e. rotation curves of galaxies, virial velocities of clusters of galaxies, and large-scale peculiar motion (deviations from uniform Hubble expansion). Evidence is mounting, but is somewhat less firm, that we live in an exactly closed universe $\left(\Omega=\rho / \rho_{\text {crit }}=1\right)$. What constitutes the dark matter is one of the outstanding scientific questions of our time. Based on calculations of primordial nucleosynthesis and the present observed isotopic abundances of light species, it is now generally agreed that ordinary baryonic matter (luminous or not) could not account for more than $10 \%$ of closure density, and thus if $\Omega>0.1$ by any appreciable amount, this would necessarily imply that a component of the dark matter involves new physics -- a previously undiscovered elementary particle, massive neutrinos, etc.

The announcement by the COBE collaboration last month of the observation of primordial fluctuations in the cosmic microwave background (and thus density Huctuations in the early universe) is of milestone significance in experimental cosmology [1-3]. The spectrum of fluctuations -- a few parts in $10^{6}$-- strongly support the hypothesis of an inflationary epoch during the big bang, which if true enforces $\Omega=1$ rigorously. Furthermore, the data prefer models where most if not all of the dark matter is non-relativistic, or 'cold dark matter' (CDM), rather than 'hot dark matter' such as massive neutrinos. These weak density perturbations grew, and seeded rapid galaxy formation which we know occurred well within a billion years after the big bang. The gravitational infall also resulted in the dark matter halos we infer observationally around all galaxies, including our own. (The present value for the critical density of the universe is about $\rho_{\mathrm{crit}} \sim 5 \mathrm{keV} / \mathrm{cm}^{3}$, which of course represents the required density averaged over the whole universe. Our local dark-matter density, best-fit to our own galactic rotation curve, is approximately $\rho$ halo $\sim 300 \mathrm{MeV} / \mathrm{cm}^{3}$.)

The axion, a light pseudoscalar particle, is one of the two most attractive cold darkmatter candidates (the other being weakly interacting massive particles). It is doubly compelling in that it was originally predicted to exist in the late 70's [4-6] as a consequence of the most minimal and elegant extension of the Standard Model to explain the strong-CP problem in QCD. Present laboratory, astrophysical and cosmological bounds limit the mass of the axion to be within $10^{-6}$ and $10^{-3} \mathrm{eV}$. The axion has two significant features relevant to experimental searches for it. The first is that its interaction with matter and radiation is proportional to its mass, and thus axions within the presently allowed window were long thought to be undetectable. The second is that the mechanism by which they would have created in the early universe (as a cold Bose-Einstein condensate) implies that the total mass of axions in the universe is inversely proportional to its mass, and thus axions of less than $1 \mu \mathrm{eV}$ would have led to an overciosure problem.

In 1983 Pierre Sikivie of the University of Florida showed that contrary to current wisdom, dark-matter axions were not so 'invisible', and in fact they could be stimulated to convert into a monochromatic microwave signal, in a high- $Q$ microwave cavity within a strong magnetic field [7]. Two small pilot experiments, one at Florida and one 
at Brookhaven, were performed but only in a narrow mass range and fell far short (101000) in the power sensitivity expected to detect dark-matter axions [8-9].

We propose to construct and operate the first experiment that would be sensitive within the expected range of axion-photon couplings, for the most important decade in axion mass $(\sim 1-10 \mu \mathrm{eV})$. This is a new proposal, which seeks to actually carry out an experimental search. A feasibility, engineering \& design study was funded at a low level by a previous LDRD award. However, this previous study has significantly refocussed our thinking on how to proceed (which initially involved the massive Axicell magnets from the MFTF-B). We now have an optimized and economical design, and have brought together a very strong collaboration with commitments for contributions of major pieces of experimental hardware.

The axion experiment consists of three elements. The first is a superconducting solenoidal magnet where the figure of merit is $B^{2} V$. The second is a series of arrays of microwave cavities, of high $Q$-factor $\left(\sim 10^{5}\right)$, each independently tunable. The third is state-of-the-art low noise cryogenic microwave amplifiers (GaAs FET's or HEMTs), and ancillary receiver electronics. LLNL is the principal among the four major institutions (LLNL, INR/Russian Academy of Sciences, MIT, and Florida), and will take responsibility for the magnet, and integration and operation of the whole experiment.

This will be a three-year proposal, where the Year I activity will be design and construction of the cryostat, testing of the coil modules, etc. In parallel with this will be the completion of the first microwave cavity assembly at INR, and the assembly and testing of the microwave electronics at MIT. In Year II the experiment will actually begin, and each of the five cavity arrays spanning the mass range $(1.6-12.6 \mu \mathrm{eV})$ will be run in roughly 6-month cycles. Support is requested for the magnet/cryostat work, part of the p.i.s' salaries, a postdoc starting in the second year, fractions of a FTE engineer, draftsman and technician, M\&S, travel, etc. The Year I request is $\$ 300 \mathrm{~K}$ in operating monies, and $\$ 50 \mathrm{~K}$ in capital equipment.

We emphasize that this experiment is a 'stand-alone' effort that will produce highvisibility physics results within the time frame of the LDRD award. It will be the first experiment with the sensitivity to possibly discover the axion. A negative result in our range of mass and coupling constant would also be scientifically important. But it would be incumbent on us to advarice both in terms of power sensitivity of the experiment (i.e. push to lower values of the axion-photon coupling), as well as in mass range upward. The discipline of actual construction and operation of the experiment would establish our credibility with the DOE Office of High Energy Physics. We anticipate their support for one of a few directions that an upgrade of the experiment might take. This could be to construct a larger, higher field magnet, or to build a dilution refrigerator for the cavities $(100 \mathrm{mK})$ and advance the state-of-the-art in effective noise of the microwave amplifiers for greater power sensitivity.

\section{The Axion}

This section is not meant to be a pedagogical discussion of the motivation and physics of the axion. A complete treatment would be lengthy and technical. Rather, this section 
will give the minimum information necessary to appreciate how the axion comes about, why the mass range of $10^{-(5-6)} \mathrm{eV}$ is prime territory for this search, and also the uncertainties involved. Two excellent reviews have appeared recently, which include all present constraints on the existence of the axion, accelerator-based experiments, and astrophysical and cosmological conclusions [10,11].

The axion is especially interesting because it responds to two fundamental problems in physics. It was predicted to exist 15 years ago as a consequence of the most simple and elegant extension to the Standard Model of particle physics to solve the "strong-CP problem". Only later was it realized that a light axion would be an excellent cold dark matter candidate.

The last remaining blemish on the theory of the strong interaction, quantum chromodynamics (QCD) is the "strong-CP problem", i.e. why the strong interaction conserves both $\mathrm{P}$ and $\mathrm{CP}$. ( $\mathrm{P}$ is parity, or reflection through the origin, and $\mathrm{C}$ is charge conjugation, or changing particles into antiparticles.) The best physical manifestation of strong-CP conservation is the absence of a neutron electric dipole moment; the present limit is $d_{\mathrm{n}}<5 \times 10^{-25} \mathrm{ecm}$. But the QCD Lagrangian contains an explicitly-CP violating piece, the so-called ' $\theta$-term'. In this term, $\theta$ is an undetermined parameter which could be any value between 0 and $2 \pi$ or multiples thereof. Only for $\bar{\theta}=\theta$ - (other unprescribed values and parameters of the theory) $=0$ is CP-violation in the strong interaction suppressed, whereas for any arbitrary value of order $O(1)$, one would expect a non-zero neutron e.d.m. of $d_{n} \sim O\left(10^{-15}\right)$ e.cm. The solution proposed by Peccei and Quinn involves postulating the existence of a global $U(1)$ symmetry for which $\bar{\theta}$ becomes a dynamical variable [4]. This symmetry is broken at some large energy scale $f_{a}$, but the effective potential for the axion field guarantees that $\bar{\theta}$ is set identically to zero, no matter what the values of the other unprescribed values and parameters in the theory. Weinberg and Wilczek realized that the spontaneous symmetry breaking of the $U(1)$ symmetry would generate a pseudo-Nambu-Goldstone boson, i.e. a physically manifest particle thereafter termed the axion [5,6]. As Peccei and Quinn had proposed (for no very good reason) that $f_{\mathrm{a}}$ should be the electroweak scale, $\sim 250 \mathrm{GeV}$, Weinberg and Wilczek predicted that the axion should have a mass of a few hundred keV, and sufficiently strong couplings to matter and radiation to permit their detection in conventional accelerator- and reactor-based experiments, nuclear decays, etc. However, such axions were quickly ruled out, and soon it was discovered how to construct variant axion models with arbitrarily large values of $f_{\mathbf{a}}[12,13]$. These were called "invisible" axion models because for $f_{a}>>250 \mathrm{GeV}$, the axion is so weakly coupled that the event rates in conventional experiments are hopelessly small.

Both the mass of the axion and all its couplings to matter and radiation are inversely proportional to the symmetry-breaking scale $f_{a}$. Thus the mass $m_{a}$ is given by

$$
m_{a}=\frac{f_{\pi} m_{\pi} \sqrt{m_{u} m_{d}}}{f_{a}\left(m_{u}+m_{d}\right)}=0.6 \mathrm{eV}\left(\frac{10^{7} \mathrm{GeV}}{f_{a}}\right)
$$

where $m_{\pi}$ is the pion mass, $f_{\pi} \sim 93 \mathrm{MeV}$ is the pion decay constant, and $m_{\mathrm{u}, \mathrm{d}}$ are the masses of the up-and down-quarks. Likewise, the coupling of the axion to two photons which is relevant to this experiment is given by 


$$
L_{a \gamma}=g_{\gamma} \frac{\alpha}{4 \pi} \frac{a}{f_{a}} F_{\mu \nu} \tilde{F}^{\mu \nu}=g_{a \gamma} a \mathbf{E} \cdot \mathbf{B}
$$

where $\alpha$ is the fine structure constant, and $g \gamma$ is a dimensionless number which depends on a parameter of the particular axion model $N_{\mathrm{e}} / N$, which can be any rational number

$$
g_{\gamma}=\frac{1}{2}\left(\frac{N_{e}}{N}-\frac{5}{3}-\frac{m_{d}-m_{u}}{m_{d}+m_{u}}\right)
$$

(We define here $\mathrm{garr}^{2}=\left(\alpha g \gamma / \tilde{\pi}_{\mathrm{a}}\right)^{2}$. As will be seen later, this quantity subsumes all of the axion physics in the expression for the microwave signal power output in the proposed experiment.)

Light weakly coupled bosons, such as the axion, are severely constrained by stellar evolution because stars would emit such particles from their whole volume, whereas they emit photons which couple more strongly to matter only from their surface. This idea has been extensively applied to the axion using a variety of stellar objects (the sun, red giants, neutron stars, white dwarfs, and recently the supernova SN 1987a), which could have produced axions by thermal processes (Primakoff production, Compton-like scattering, axion bremsstrahlung in electron-nucleon and nucleon-nucleon scattering, etc.). Reference [11] gives an excellent discussion of this method. The supernova SN $1987 \mathrm{a}$ establishes the strictest bound on the axion mass, $m_{\mathrm{a}}<10^{-3} \mathrm{eV}$; axions between 1 $\mathrm{meV}$ and about $1 \mathrm{eV}$ would have resulted in a dramatic foreshortening of the neutrino burst detected in both the IMB and Kamiokande large water Cerenkov detectors.

The cosmological production of axions implies a lower limit on the order of $10^{-6} \mathrm{eV}$. This limit follows from the requirement that the present cosmological axion density not exceed the critical density for closing the universe. When the universe cooled below a critical temperature of order $f_{a}$, the spontaneous breaking of the $U_{\mathrm{PQ}}(1)$ symmetry would result in an initial misalignment angle of the axion field at each point in space . When the temperature further cooled below the QCD phase transition, non-perturbative QC.D effects would generate an effective potential in $\bar{\theta}$ endowing the axion with a mass, and the axion field will begin to oscillate about the CP-conserving direction, $\bar{\theta}=0$. The energy density stored in these axion field oscillations today approximately equals

$$
\rho_{a}^{\mathrm{vac}}\left(t_{0}\right) \approx \rho_{c r i t}\left(t_{0}\right)\left(\frac{0.6 \cdot 10^{-5} \mathrm{eV}}{m_{a}}\right)^{7 / 6}\left(\frac{200 \mathrm{MeV}}{\Lambda_{Q C D}}\right)^{2}\left(\frac{75 \mathrm{~km} / \mathrm{s} \cdot M p c}{H_{0}}\right)^{2}
$$

where $\rho_{\text {crit }}\left(t_{0}\right)$ is the present critical energy density for closure of the universe, $H_{0}$ is the present value of the Hubble constant and $\Lambda_{Q C D}$ is the QCD scale factor.

There are significant uncertainties in this estimate and the corresponding lower bound on the axion mass, which we will take hereafter nominally as $1 \mu \mathrm{eV}$ to account for the uncerlunties in both the Hubble constant and QCD scale factor. However, there are uncertainties beyond purely numerical ones. These involve e.g. whether inflation occurred before or after the $P Q$-phase transition and the details of the inflationary scenario; possible entropy release in the early universe; and possible axion radiation from cosmic axion strings. The point of view proper to experimental physics is that one 
should ultimately plan for a full exploration of the three decades in mass range open to the axion. Our present point of view however is that the decade $\sim(1-10) \mu \mathrm{eV}$ corresponding to closure density in axions under the standard vacuum realignment mechanism is the most exciting place to start, and it is precisely here which is immediately feasible experimentally.

\section{The Cavity Microwave Detector for Galactic Halo Axions}

As shown by Pierre Sikivie in 1983, axions which constitute our own galactic darkmatter halo could be detected by their stimulated conversion to microwave photons in a microwave cavity inside a strong magnetic field [7]. Such axions are non-relativistic, having only the virial velocity of the galaxy, $\beta \sim 10^{-3}$, and thus their energies have a spread only of order $10^{-6}$ above their rest mass. As axions couple to two photons, a Primakoff process in a static external potential (the $B$-field) yields one real photon of energy $m c^{2}\left(1+O\left(\beta^{2}\right)\right) \sim m c^{2}\left(1+O\left(10^{-6}\right)\right)$.

The realization of this technique in an experiment consists of three major components, as shown in Figure 1. The first component is a magnet, with the largest value of $B^{2} \mathrm{~V}$ achievable. Most practically this is a superconducting solenoid. The second component is one or more tunable microwave cavities with a high loaded quality factor, $Q_{L}$. (At the magnetic fields we wish to use, of order 10 Tesla, superconducting cavities are not an option, so one must use cavities made of oxygen-free high conductivity copper or possibly very pure aluminum.) The third component is state-of-the-art ultra-low noise cryogenic microwave amplifiers, and the other receivor and data acquisition electronics. The cavity or array of cavities is tuned, and the conversion probability for axions to photons goes through a sharp maximum when $h v=m_{\mathrm{a}} c^{2}$. A frequency of $1 \mathrm{GHz}$

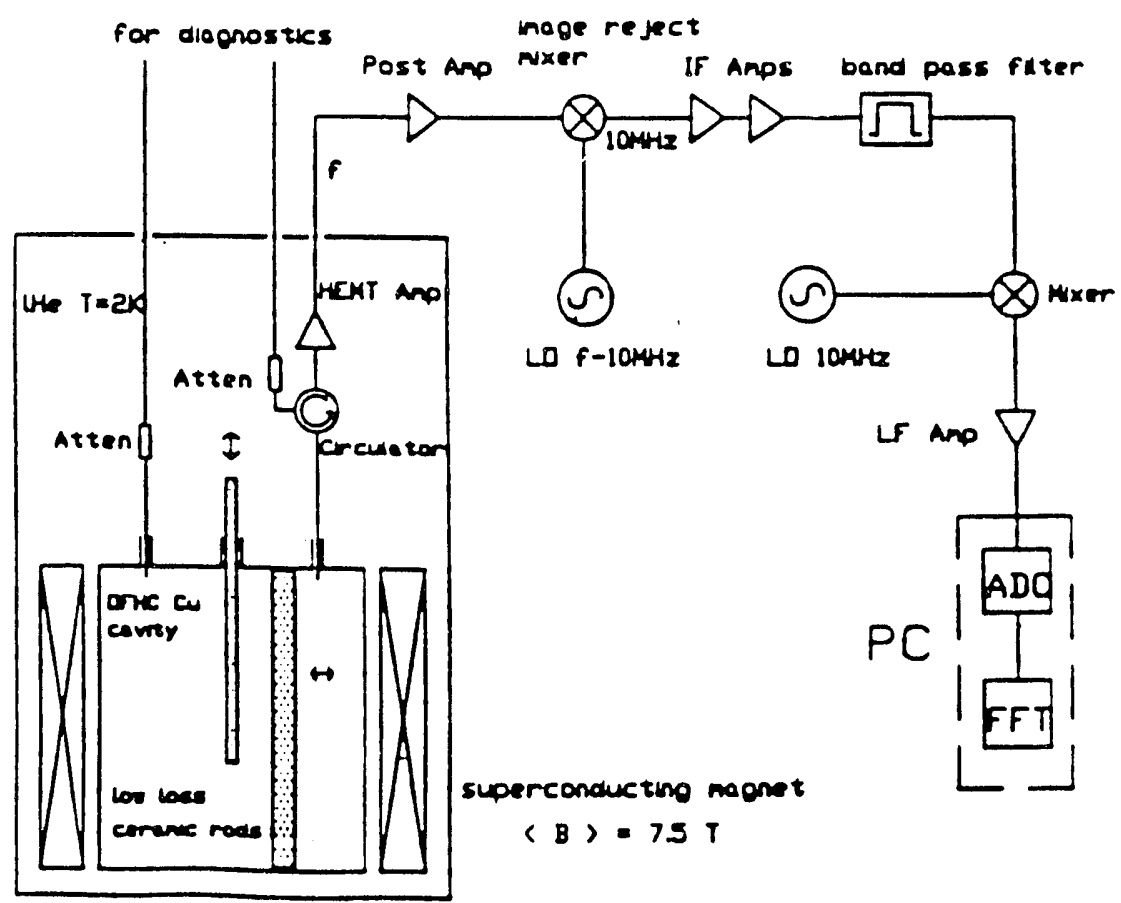

Figure 1. Schematic diagram of the University of Florida pilot axion experiment [14]. 
corresponds to an axion of mass $4.136 ! ! \mathrm{eV}$, and thus in this experiment we are interested in the range of frequencies from below $400 \mathrm{MHz}$ to above $3 \mathrm{GHz}$.

As seen in Eqn. (2), the interaction Lagrangian which couples the axion to two photons is an E-B interaction. This implies that for a right-circular cavity placed in the bore of the solenoidal magnet, only the $\mathrm{TM}_{\text {nlo }}$ modes couple axions to photons. In fact, the only really practical mode is the $T_{010}$ mode, for which the 'form factor' of the mode $C_{n l}$ (the overlap integral between the $B$ - and $E$-fields) achieves a theoretical maximum of 0.69 . (It is also useful to keep in mind that the frequency of the $\mathrm{TM}_{010}$ mode is given by (115 $\mathrm{MHz} / R[\mathrm{~m}]$ ), where $R[\mathrm{~m}]$ is the cavity radius in meters; obviously the bore of the magnet sets a lower limit to the mass of the axion that one may search for.) The power on resonance for the axion-photon conversion is given by

$$
\begin{aligned}
& P_{n l}=\left(\frac{\alpha}{\pi} g_{y} \frac{1}{f_{a}}\right)^{2} V B_{0}^{2} \rho_{a} C_{N} \frac{1}{m_{a}} \operatorname{Min}\left(Q_{L}, Q_{a}\right) \\
& P_{n l}=4 \cdot 10^{-26} \text { Watt }\left(\frac{V}{0.22 \mathrm{~m}^{3}}\right)\left(\frac{B_{n}}{10 \text { Tesla }}\right)^{2} C_{n l}\left(\frac{g_{y}}{0.97}\right)^{2} . \\
& \left(\frac{\rho_{a}}{\frac{1}{2} \cdot 10^{24} \mathrm{gr} / \mathrm{cm}^{3}}\right)\left(\frac{m_{a}}{2 \pi(1 \mathrm{GHz})}\right) \operatorname{Min}\left(Q_{L}, Q_{a}\right)
\end{aligned}
$$

where $V$ is the volume of the cavity, $B_{0}$ the r.m.s. magnetic field strength along axis, $\rho_{a}$ the density of galactic halo axions at earth, and $Q_{a} \sim 10^{6}$ is the "quality factor" of the galactic halo axions, i.e. the ratio of their mass to their energy spread.

In a real experiment, what is important is not only the conversion power, but the noise as well. The total noise is given by $T_{n}=T_{\text {elec }}+T_{\text {phys, }}$, where the two components represent the intrinsic electronic noise temperature, and the physical bath temperature. In this proposal, the magnet cryostat is being designed for operation at a physical temperature of $1.8 \mathrm{~K}$. Concerning the electronic noise, the very best low-noise microwave amplifiers in the GHz range are GaAs HEMT's (high electron mobility transistor), with an equivalent noise temperature of about $1-2 \mathrm{~K}$ at $1 \mathrm{GHz}$, and increasing $1 \mathrm{~K}$ per $\mathrm{GHz}$. Below $600 \mathrm{MHz}$, GaAs FETs are the best.

The search rate of the experiment is given by

$$
\begin{aligned}
& \frac{d f}{d t}=\frac{72 G H z}{\text { year }}\left(\frac{4}{s / n}\right)^{2}\left(\frac{V}{0.22 m^{3}}\right)^{2}\left(\frac{B_{0}}{10 \text { Tesla }}\right)^{4} C^{2}\left(\frac{g_{\gamma}}{0.97}\right)^{4} . \\
& \frac{\rho_{a}}{\frac{1}{2} \cdot 10^{-24} \mathrm{~g} / \mathrm{cm}^{3}}\left(\frac{5 K}{T_{n}}\right)^{2}\left(\frac{f}{1 G H z}\right)^{2}\left(\frac{Q_{w}}{Q_{a}}\right)
\end{aligned}
$$


where $(s / n)$ is the desired signal-to-noise ratio for detection of the axion (or equivalently exclusion of an axion at the $97.7 \%$ c.1.), and $Q_{w}$ is the 'wall' or unloaded $Q$ of the cavity. (The loaded $Q$ is given by $1 / Q_{L}=1 / Q_{w}+1 / Q_{\text {out }}$, where $Q_{\text {out }}$ is the contribution due to the output coupler. The expression above assumes that the search rate is maximized by the optimization $Q_{L}=Q_{W} / 3$.) With the benchmarks for the proposed experiment, we may rewrite this in a more practical form

$$
\frac{1}{f} \frac{d f}{d t}=\frac{6.4 \%}{\text { week }}\left(\frac{4}{s / n}\right)^{2}\left(\frac{B_{n}}{10 \text { Tesla }}\right)^{4} P^{2} C^{2}\left(\frac{5 K}{T_{n}}\right)^{2}\left(\frac{f}{1 G H z}\right)\left(\frac{Q_{w}}{10^{5}}\right)\left(\frac{1}{N_{\text {rescan }}}\right)\left(\frac{\Delta f}{\delta f}\right)
$$

where $P$ is the packing fraction of cavities filling the magnet bore, $N_{\text {rescan }}$ is the average number of times a given region is automatically rescanned due to showing a $2 \sigma$ peak, $\Delta f$ is the frequency interval between successive spectra, and $\delta f=f / Q L$ is the cavity bandwidth. We have assumed an axion-photon coupling of one particular model, indicated later as $\operatorname{KSFZ(a)~[13],~and~that~axions~saturate~the~halo~density~of~} 300$ $\mathrm{MeV} / \mathrm{cm}^{3}$.

In the late 1980's, two pilot experiments were performed, one at BNL by a RochesterBrookhaven-Fermilab collaboration [8], and one at the University of Florida [9]. We give here a brief description of the Florida experiment, which was the PhD thesis work of Chris Hagmann, presently at UC Berkeley [14]. Our proposed experiment draws more closely from the experience and design of the UF experiment, and in fact they are our collaborators on this proposal. The magnet in that experiment was $40 \mathrm{~cm}$ long with an inner diameter of $17 \mathrm{~cm}$. The volume-averaged field over the $0.008 \mathrm{~m}^{3}$ volume was 7.5 Tesla. Some of the experimental runs were carried out at a physical temperature of $4.2 \mathrm{~K}$, and others at $1.8 \mathrm{~K}$. Unloaded-Q's of 150,000 were achieved below $20 \mathrm{~K}$ in the two cavities that were used. As the axion signal that is being sought is narrower by around a factor of 20 from the bandwidth associated with $Q \mathrm{~L}$, a power spectrum of 32 channels was measured around each central frequency of the cavity. Coarse tuning of the cavities was accomplished by radial displacement of either a dielectric or metal rod with respect to the cavity axis (allowing one to go down or up by roughly $25 \%$ in frequency with respect to an empty cavity, without undue diminution of $C^{2} Q$ ). Fine tuning was accomplished by longitudinal insertion of a small dielectric rod by a stepping motor and gear reduction. A detailed discussion of various conceptual designs, electromagnetic properties, required tolerances, etc. associated with high- $Q$ cavity design and tuning for the axion experiment, along with actual cavity data is found in ref. [15].

The signal from the microwave amplifier was mixed down twice to the audio frequency range $(0-32 \mathrm{kHz})$, digitized, and the power spectrum calculated real-time by a Fast Fourier Transform (FFT) board in the PC, which does both data acquisition and analysis, as well as complete automated control of the experiment. The signal was sampled at a $70 \mathrm{kHz}$ rate (as required by Nyquist's theorem), and 64 successive samples measured and averaged to produce one spectrum. In a 90-second run at a given frequency, $10^{5}$ spectra are acquired and averaged. If no peak above $2 \sigma$ shows up, the $P C$ steps the frequency of the cavity, measures the central frequency and $Q$ of the cavity, and begins the next run. If a peak above $2 \sigma$ shows up, another $10^{5}$ spectra are taken and averaged with the first. If the peak remains statistically significant after a 

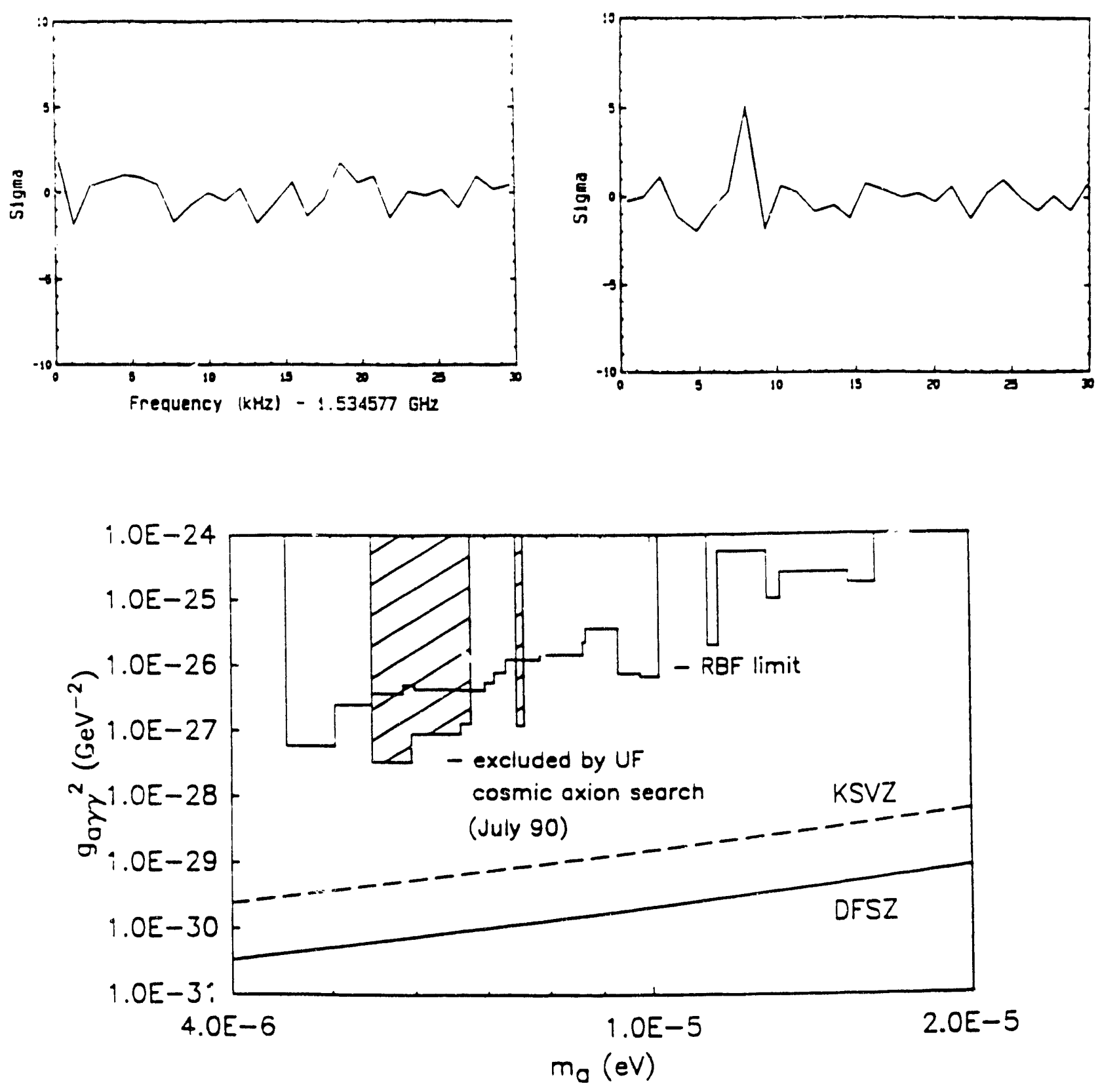

Figure 2. (Top left) Typical power spectrum from the UF experiment, showing no peaks exceeding $2 \sigma$. (Top nght) Spurious peak, due to a harmonic of the $16 \mathrm{MHz}$ clock frequency from the PC. (Bottom) The $\mathrm{RBF}[8]$ and $U F[9,14]$ exclusion regions in the mass vs. axion-photon coupling constant plane. 
maximum of 5 tries, the peak is flagged for later investigation, and the experiment continues. Figure 2 top shows two spectra from the UF experiment. One is a spectrum without a candidate peak, and the other shows on - of the rare candidate peaks which were dete ted during the entire course of the project. The beauty of the cavity microwave technique is that a real axion peak can be verified unambiguously -- when the magnetic field is turned off, the signal should go away, and the power associated with a real signal would vary as $B 2$. Obviously no peak in either the UF or RBF pilot experiments satisfied that criteria. The spuriolis peak (Fig. 2, top right) was associated with a harmonic of the $16 \mathrm{MHz}$ clock frequency of the computer. Figure 2 (bottom) shows the UF and RBF exclusion regions with respect to two axion models. The solid line labeled DFSZ [12] corresponds to a model with generic axion couplings to all fermions, but has a relatively unfavorable axion-photon coupling. The dashed line labeled KSVZ [13] corresponds to one particular choice of PQ charges within the 'hadronic' class of axions, i.e. those with no tree-level coupling to leptons or quarks, except to heavy, possibly yet-undiscovered quarks. It is provocative that for an equally plausible choice of $\mathrm{PQ}$ charges in a $\mathrm{KSVZ}$ axion (shown later), the pilot experiments would have very nearly had the power sensitivity for its detection [16]!

\section{The Proposed Experiment}

This section is a description of the experiment proposed for LDRD funding in FY 1993. A great deal of experimental detail is not warranted here as the proposed experiment, with one exception, is largely a scale-up of the UF pilot experiment which is fully described in the references below. Rather, we will emphasize the advances we have made which now make a major assault on the axion possible. We will also demonstrate that we have a critical mass of personnel, and key capital commitments for both microwave cavities and the microwave electronics.

\section{The NASA SUMMA magnets}

The easiest and most obvious way to reach into the cosmologically significant region of axion-photon coupling is a scale-up of $B^{2} V$ of the magnet. For a long time we studied the feasibility of the use of the remaining pair of Axicell magnets from the decommissioned MFTF-B ( $3 \mathrm{~m}^{3}, 7$ T field), which would have given us an immediate factor of 300 improvement in power sensitivity over the pilot experiments. However, the sheer size of the magnets (50 tons) proved prohibitive in both initial cost to remove them and set them up in their own vessel (at least $\$ 2.5 \mathrm{M}$ ), and operating cost ( $\$ 250 \mathrm{~K} /$ year for helium and power).

We have made two great discoveries in the magnet for the axion search. The first is the NASA SUMMA coils, presently at the University of Missouri, which Prof. Mark Prelas has kindly made available for us to bring here for the purposes of this experiment. There are three coils, and each $35 \mathrm{~cm}$ coil consists of three modules, an inner $\mathrm{Nb}_{3} \mathrm{Sn}$ module, and middle and outer modules of NbTi. The three NASA SUMMA coils (Superconducting Magnetic Mirror Apparatus) put together make a very compact but extremely high field magnet $(50 \mathrm{~cm}$. clear-bore i.d., $1.1 \mathrm{~m}$ depth, possibly 10.5 Tesla at 


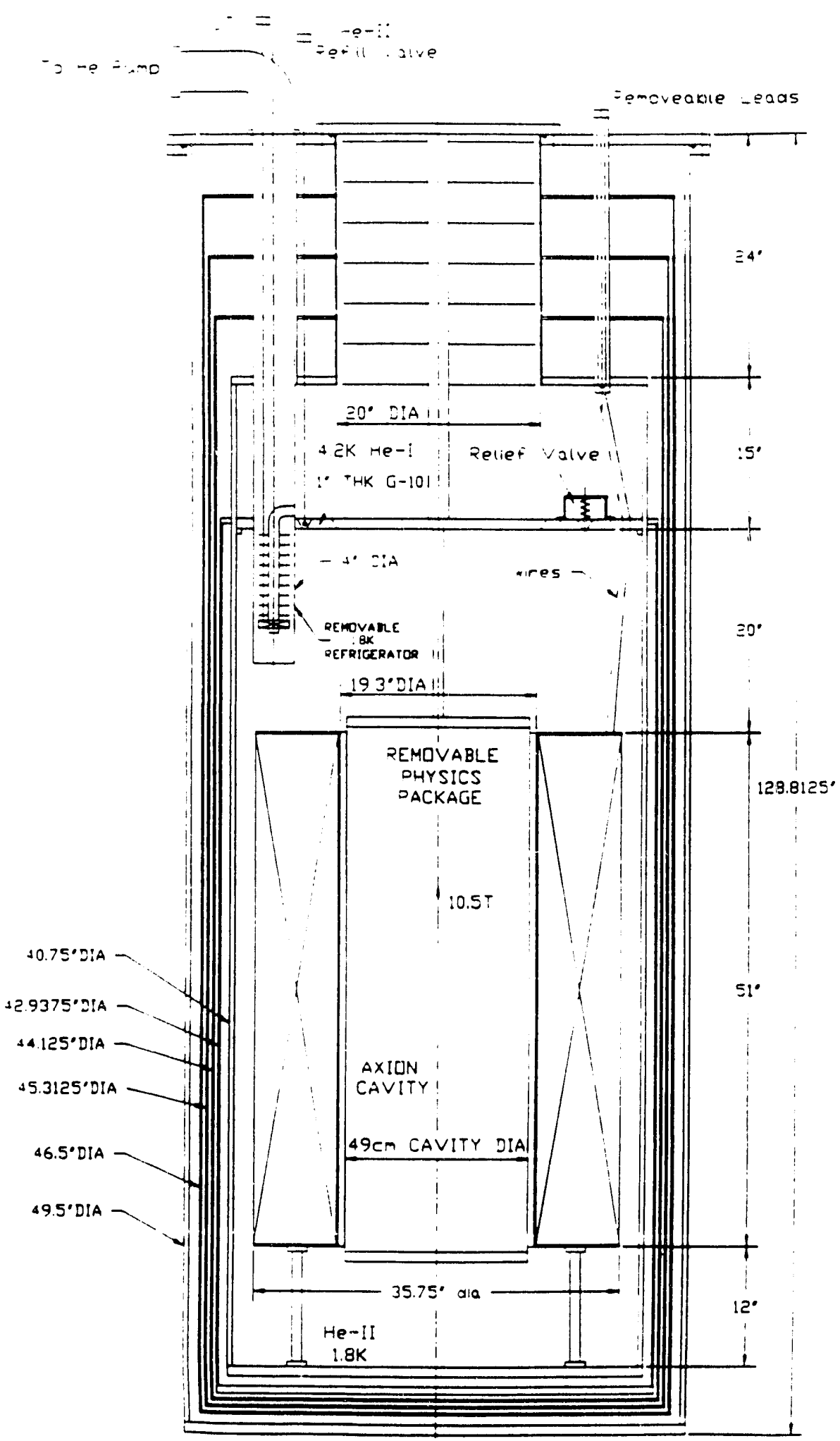

Figure 3. Design of a thermally efficient cryostat, and $1.8 \mathrm{~K}$ refrigerator for the NASA SUMMA coils. 
$1.8 \mathrm{~K}$ ), weighing only 4.5 tons. Along with the magnet comes all the power supplies, control hardware, and high quality spare superconducting wire.

The NASA SUMMA magnet gives us an immediate factor of 50 over the UF pilot experiment, before counting any incremental improvements in HEMT amplifiers, etc..

The second breakthrough has been the involvement of an industrial partner, Wang NMR of Livermore. Sou-Tien (Bert) Wang is one of the premier superconducting magnet builders in the world, having built more than 70 in his career at Argonne and at LLNL. (He was a key person in the design and construction of the MFTF-B.) Now principally in the medical NMR business, he performed a study for us resulting in a very economical and thermally efficient cryostat and $1.8 \mathrm{~K}$ refrigerator design. Operating the coils in persistent mode with removable leads, and bringing bo bear medical NMR cryostat technology to our experiment, it is estimated that the helium consumption would be no more than about 3.5 liters/ hour even if all the mechanical motion was transmitted to the tuning mechanism of the cavity arrays with s.s. tubing; if $\mathrm{G}-10$ is used, the helium consumption might only be $350 \mathrm{ml} /$ hour. Figure 3 shows the design of the cryostat, magnet and $1.8 \mathrm{~K}$ retrigerator.

The timeline for delivering a complete system is one year, and will cost $\$ 400 \mathrm{~K}$. This includes all transportation of the magnet components from Columbia, Missouri, fabrication of the cryostat, persistent switch, etc., testing of the coils, and refurbishing of the power supplies and control system.

Bert is also a high energy physicist, and will be a collaborator on the experiment. He has offered to us his new plant in which to actually run the experiment, should it be convenient to do so. Wang NMR is located on North Canyons Parkway, just across I580 from the Livermore airport, about 10 minutes drive away. We are considering this seriously, as we have Russian collaborators for whom it might be much easier to have work there than within the Lab, where they need to be escorted continuously.

\section{The INR Microwave Cavities}

As one searches for axions higher in mass, the radius of the microwave cavities goes down as the inverse of the axion mass. Therefore to take maximum advantage of the $B^{2} V$ of the magnet it is essential to use arrays of cavities, each cavity being independently tunable, and whose outputs are power-combined. (This is distinct from coupling cavities, however.) Neither of the pilot experiments ever ran with more than one cavity. As a final chapter to his thesis, however, Chris Hagmann successfully demonstrated the principle of combining the output from two cavities in a test at $300 \mathrm{~K}$. The magnitude and shape of the $Q$-curve of the combined cavities was in exact agreement with theory. Nevertheless, the fabrication and efficient operation of arrays of many combined cavities was perceived to be the major obstacle to mounting a full-scale experiment.

A year ago, a strong group from the Institute for Nuclear Research of the Russian Acaderny of Sciences headed by Dr. Nikolai A. Golubev joined our collaboration, proposing that they take complete responsibility for providing all the microwave 


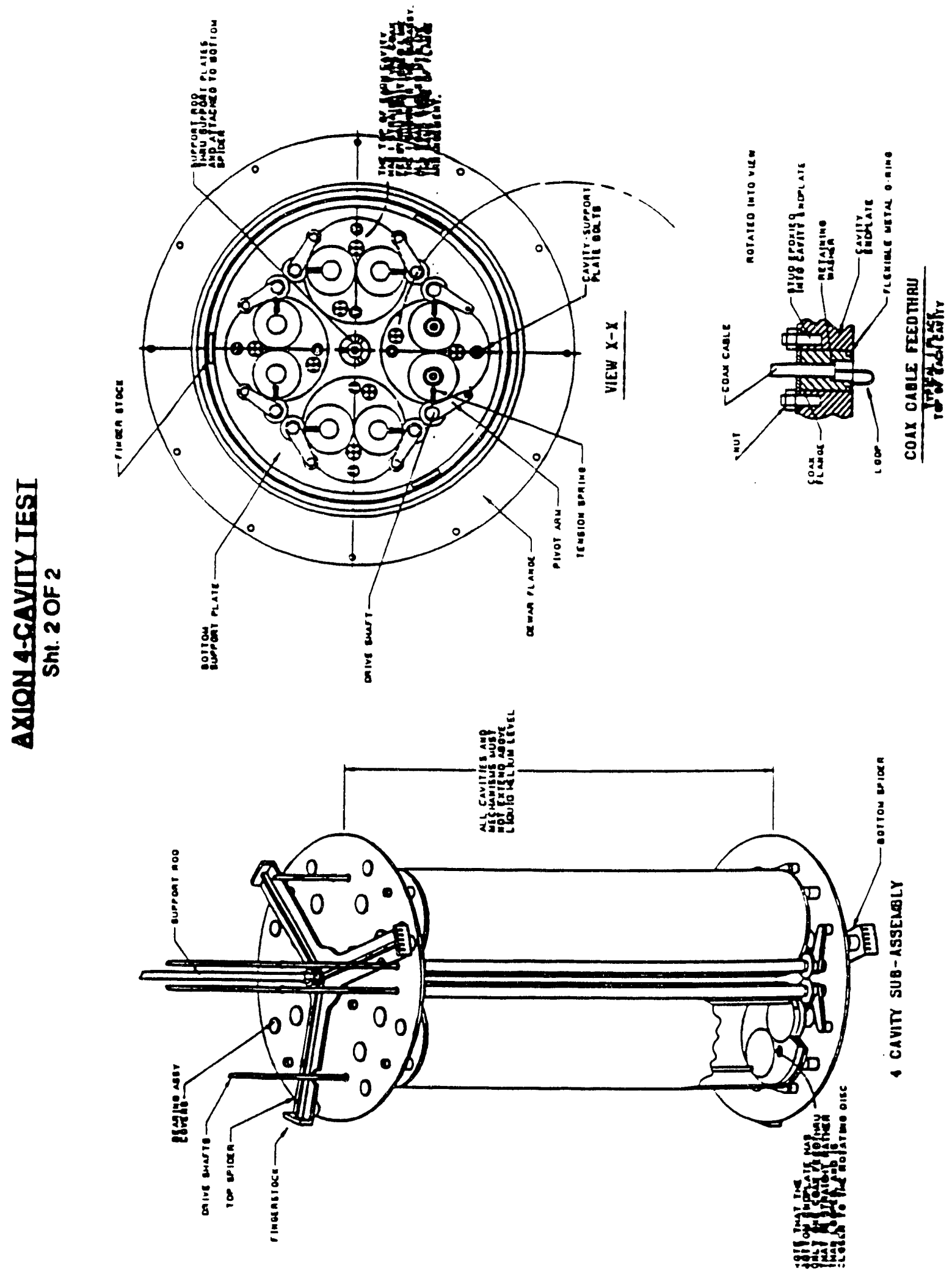

Figure 4. Conceptual design of the INR 4-cavity array. Total diameter $460 \mathrm{~mm}$; length $900 \mathrm{~mm}$. 
cavities for the experiment. Furthermore, they suggested a novel technology for fabricating the cavities, namely metalized precision-formed ceramic. Ceramic cavities are light, strong and require a minimum of machining after being fired to hold mechanical tolerances. INR has long experience in metalized ceramics, principally for beam pipe in rapid-cycling synchrotrons.

During a visit to the INR laboratories in Troitsk, in October, 1991, a Memorandum-ofUnderstanding was written between INR, LLNL and the University of Florida for R\&D on metalized ceramic cavities. Basically, INR agreed to provide both full-size single cavities (to test different coatings, e.g. Al, $\mathrm{Cu}$, and metalization techniques) and a 4 cavity array (to test the automated tuning of multiple cavities). Figure 4 shows an approximate engineering drawing of the 4-cavity prototype array. The ceramic components are being made at the Lutch aircraft company in Kharkov, and the magnetron sputtering is done by the Zinit optical company in Zilinograd. The results at $300 \mathrm{~K}$ are encouraging. The measured $\mathrm{Q}$ in the $\mathrm{TM}_{010}$ mode for one of their large cavity is 39,000 compared to a theoretical maximum of 43,600 . However, only by testing the cavities at LHe temperature can one confirm the quality of the coating.

Three INR physicists and engineers will visit LLNL for the month of September, where the 4-cavity array will be tested at $4.2 \mathrm{~K}$ in a $50 \mathrm{~cm}$ i.d. LHe dewar in the MFE cryogenics test lab. This array is already of the full size that would be used in our actual experiment in the NASA SUMMA magnet. They will spend October at the University of Florida, testing magnetoresistive effects at $4.2 \mathrm{~K}$ with single cavities in the magnet and dewar used in the UF pilot experiment.

The experiment will require five arrays to cover the mass range proposed. The first is a single large cylindrical cavity. The second is large cylinder partitioned into two cavities of semicircular cross section. The third is the 4-cavity array presently under construction. The fourth and fifth are 8- and 16-fold close-packed arrays. Details on the design of an experiment with a large number of cavities may be found in ref. [17].

The Vice-Director of INR, Dr. Valery Rubakov has sent a letter to Bruce Tarter confirming our understanding that they would provide all the cavities required, tuning mechanisms, etc. should this experiment be approved and funded.

\section{The microwave electronics}

The extreme weakness of the signal being looked for puts a premium on state-of-the-art ultra-low noise microwave amplifier technology. The collaboration is strong in this area, both in manpower and in capital commitments.

A key collaborator in this experiment will be Prof. Leslie Rosenberg, who joined the effort only a few months ago. He is one of the best young high energy physicists in the country, and most recently was responsible for most of the design and construction of the electronics and data acquisiton system for the CASA experiment (Chicago Air Shower Array) in Dugway, Utah. He has recently accepted an offer to join the faculty at MIT, with the expressed intention of working solely on the axion search for his first term there. Effectively he is staking his promotion to tenure on this experiment. The Department, Dean and Provost have been extremely supportive of his intentions. MIT 
is giving him approximately $\$ 300 \mathrm{~K}$ in capital equipment money to build up the entire microwave electronics for the experiment, and about $\$ 60 \mathrm{~K}$ in operating money. This is of course contingent on the experiment being approved and funded this year by LLNL.

Frank Nezrick of FNAL, one of the two principal senior people in the Rochester-BNLFNAL pilot cavity microwave experiment has also recently joined us. He brings long experience in microwave techniques, as well as microwave electronics (synthesizer, local oscillator, network analyzer, etc.) used in the RBF experiment.

Walter Ferguson of MFE is our own local expert in microwave electronics, and has begun characterizations of a copper-plated s.s. 3-GHz prototype cavity that was fabricated by our UCB/LBL collaborators a year ago. Francesco Villa of SLAC is also a great resource.

\section{Other resources and personnel}

Chris Hagmann at Berkeley, and Dave Tanner and Neil Sullivan at Florida have overall experience in the cavity axion experiment. We will make use of the free machine shop time at Florida to do most of our shop work. For example, each cavity array will require a custom-made flange for the top of the cryostat from which it will be suspended, and through which all mechanical and electrical penetrations are made. It is possible that we will be able to make use of both FNAL, and LBL through our collaborator Dennis Moltz, for limited technical support as well.

\section{Discussion}

\section{Scientific deliverable}

Figure 5 shows the region in axion mass and axion-photon coupling where galactic halo axions would either be discovered $(s / n>4)$, or excluded $(97.7 \%$ c.l.) by our experiment. The actual limit will probably be somewhat better due to improvements in GaAs HEMT amplifiers even in the past two years. The nominal axion mass for closure of the universeis $6 \mu \mathrm{eV}$, assuming that the standard vacuum realignment mechanism dictates the arion contribution, but as previously mentioned there are large uncertainties here. The three model lines (DFSZ, and two choices of parameters for KSVZ axions) give a fair representation of our uncertainty in how strongly the axion couples to photons.

\section{Strategy}

This experiment will make a factor of 100 improvement in power sensitivity over the pilot experiment, and cover a much broader mass range. It would be the first experiment with cosmologically significant sensitivity, although it is clear that at present we could not exclude a DFSZ axion. Nevertheless, theory should never be taken too seriously. Whenever one can make even an order-of-magnitude improvement in an experiment, or reach into the physical region of interest for the first time, it is imperative to do so as soon as possible. 


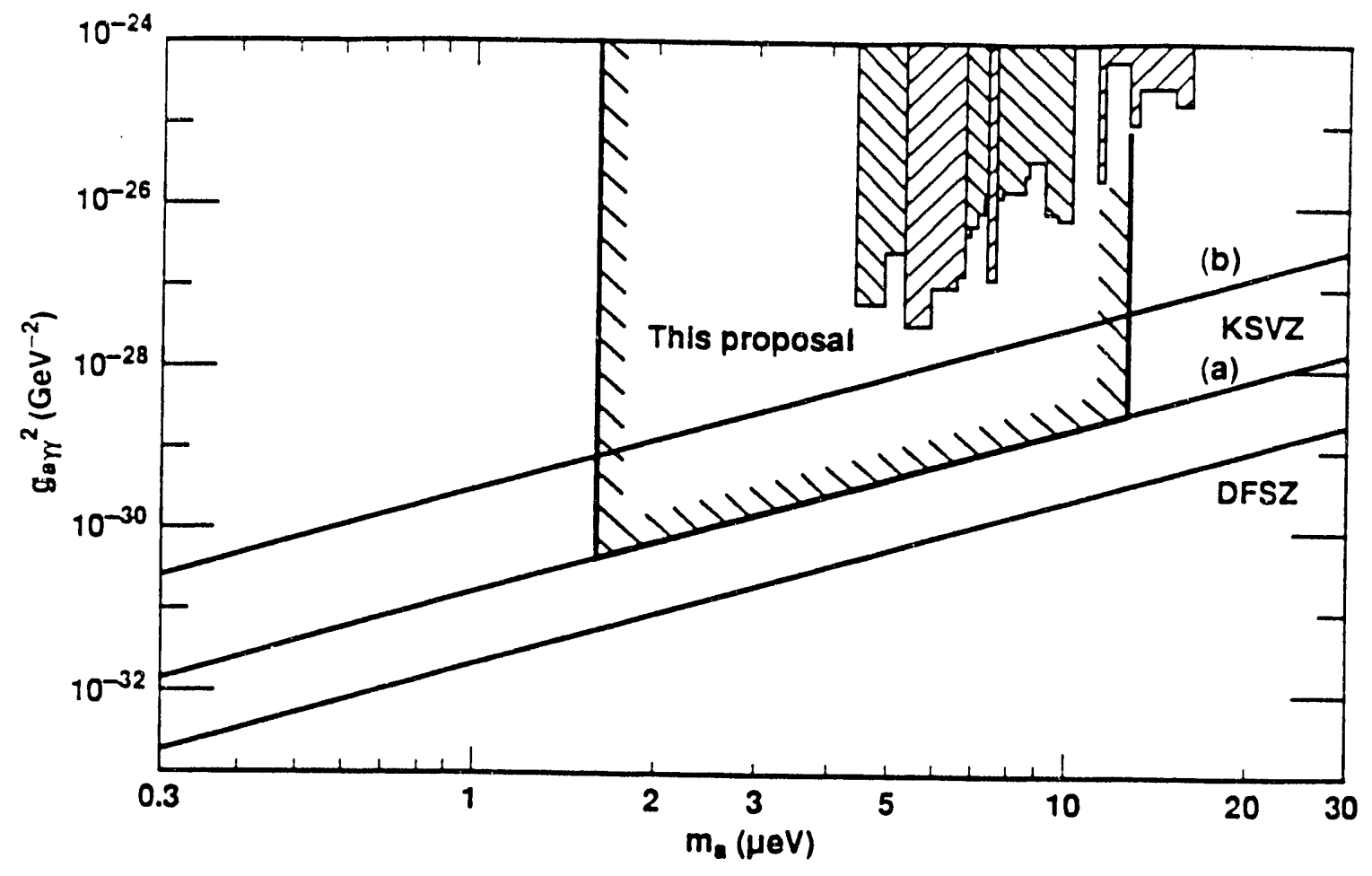

Figure 5. Region of mass and axion-photon coupling that the proposed experiment will be sensitive to.

Our strategy of an experiment with a compact high field magnet, and emphasis on as low a total noise as possible is driven by three motives. (i) The first and most important is the reason of timeliness as mentioned above. Our experiment is small enough (physically and budgetarily) that we will be taking data one year after starting. There has been a great awakening of interest in the dark-matter problem within the past year, even within the past months. While we have no competition at present, it is unlikely that situation will pertain forever. A third pilot effort existed at KEK in Japan, but was discouraged from proceeding to a large-scale experiment at that time due to budgetary pressures resulting from the Japanese B-Factory project. A Kyoto-Osaka group was engaged in advanced receiver technology for the axion search. (ii) The second reason is that we envision the way to push this experiment in the future is by advancing on the front of low total noise (the quantum limit at $1 \mathrm{GHz}$ is $50 \mathrm{mK}$ ). We have depth in this area and are anxious to begin R\&D on customized HEMT's, possibly maser amplifiers, etc.. But this requires lowering the physical temperature (i.e. by a dilution refrigerator) in parallel with reduction in electronic noise, and that is easier for a smaller rather than a larger detector. (iii) The third is that we will also want to continue upward in mass, which will involve progressively smaller cavity structures. Trading off high field vs. volume (and one can make an insert booster coil to raise the field higher still) minimizes the number of cavities one needs to deal with. 


\section{Future funding}

Should the axion not be found in this experiment, we will want to advance both in power sensitivity (and thus downward in $g a \gamma^{2}$ ), and upwards in mass. Both will depend on insights as we go along. From several conversations with the DOE Office of High Energy Physics over the past two years, we know they are very interested in a coherent effort towards finding or excluding the axion. We believe DOE would be receptive to a proposal for an upgrade, particularly one that emphasized advances in ultra-low noise microwave electronics. P.K. Williams of DOE has already indicated to Les Rosenberg that he would encourage submission of an R\&D proposal immediately. Also, once the experimental 'platform' is established, future running of the experiment should be very economical, and this we could seek from DOE as well.

It is reasonable to ask why DOE/HEP would not fund the full construction of the experiment here from the outset. Beyond the usual sensitivities concerning ER putting monev into a DP lab in competition with support of university groups, there is a more pressing issue. The DOE Office of High Energy Phvsics requested guidance from the High Energy Physics Advisory Panel (HEPAP) on how to manage the 'base program' over the coming decade in the face of enormous fiscal pressures due to the SSC. HEPAP in turn charged a subpanel headed by Prof. Michael Witherell of UCSB to carry out this study. Their report, submitted this April, was not encouraging with respect to growth in the area of non-accelerator particle physics, or anything else for that matter. In fact, the projections for FY93 indicate a $20 \%$ decrease from FY92 in non-accelerator physics from what was only a $\$ i j M /$ year portfolio.

\section{Important Remark}

We believe that we have an optimized concept for the axion experiment, and that the time has never been more ripe to launch the effort. The project has the great benefit of very substantial commitments of manpower and capital resources, particularly the offer by the Institute of Nuclear Research of the Russian Academy to provide all of the microwave cavities, and the offer by Prof. Rosenberg of MIT of $\$ 300 \mathrm{~K}$ in microwave electronics. However, it is equally clear that if this LDRD proposal is not approved this year, both these commitments will evaporate, and the opportunity for LLNL to play a leadership role in the search for the axion will be permanently lost.

\section{Budget and Timeline}

\section{FY93 Budget}

The budget request for FY93 is $\$ 302 \mathrm{~K}$ in operating money, and $\$ 50 \mathrm{~K}$ in capital equipment, as detailed below (in $\$ \mathrm{~K}$ ).

\section{Operating:}




\begin{tabular}{lrl} 
Manpower Total & 82.2 & 0.25 KVB, 0.25 (M.E. + Des.) \\
Procurements & 205.0 & Principally Wang NMR \\
Travel (Foreign) & 3.0 & Support of INR visitor, 1 mo. \\
Travel (Domestic) & 1.0 & \\
Stores & 1.0 & \\
TID & 1.0 & \\
Lab. Gen'l. Exp. & 8.8 & \\
\hline PROJ. OP. TOTAL & 302.0
\end{tabular}

Capital:

Fluke Local Oscillator (100 MHz-2 GHz) $\quad 13.0$

Fluke Local Oscillator $(10 \mathrm{MHz}) \quad 14.0$

H-P Power Meter $\quad 8.0$

H-P RF Switch $\quad 7.0$

Berkshire Cryogenic Amplifier $\quad 8.0$

$\begin{array}{ll}\text { PROJ. CAP. TOTAL } & 50.0\end{array}$

\section{Timeline}

The major milestones for the cryostat work by Wang NMR are shown in Figure 6.

The major milestones for the cavity work by INR are:

(1) Completion/reporting prototype tests at LLNL, UF 9-10/1992

(2) Fabrication of first array ( $460 \mathrm{MHz}$ central freq., single cavity)

(3) Transport to LLNL

The major milestones for the microwave electronics work at MIT are:

(1) Establishment of lab space

(2) Procurement of all receiver components

(3) Assembly and testing with $3 \mathrm{GHz}$ prototype cavity

(4) Writing data acquisition and analysis software

(5) Transport to LLNL 
The major milestones for integration and project oversight at LLNL are:

(1) Engineering and design of the flange for the first cavity array

(2) Machining of the flange at UF or FNAL

(3) Writing experiment control software

(4) Integration of cavity/tuner \& cryogenic amp on flange

The longer term timeline is shown in Figure 7 , indicating six-month run periods with one cavity array in parallel with the preparation of the next array.

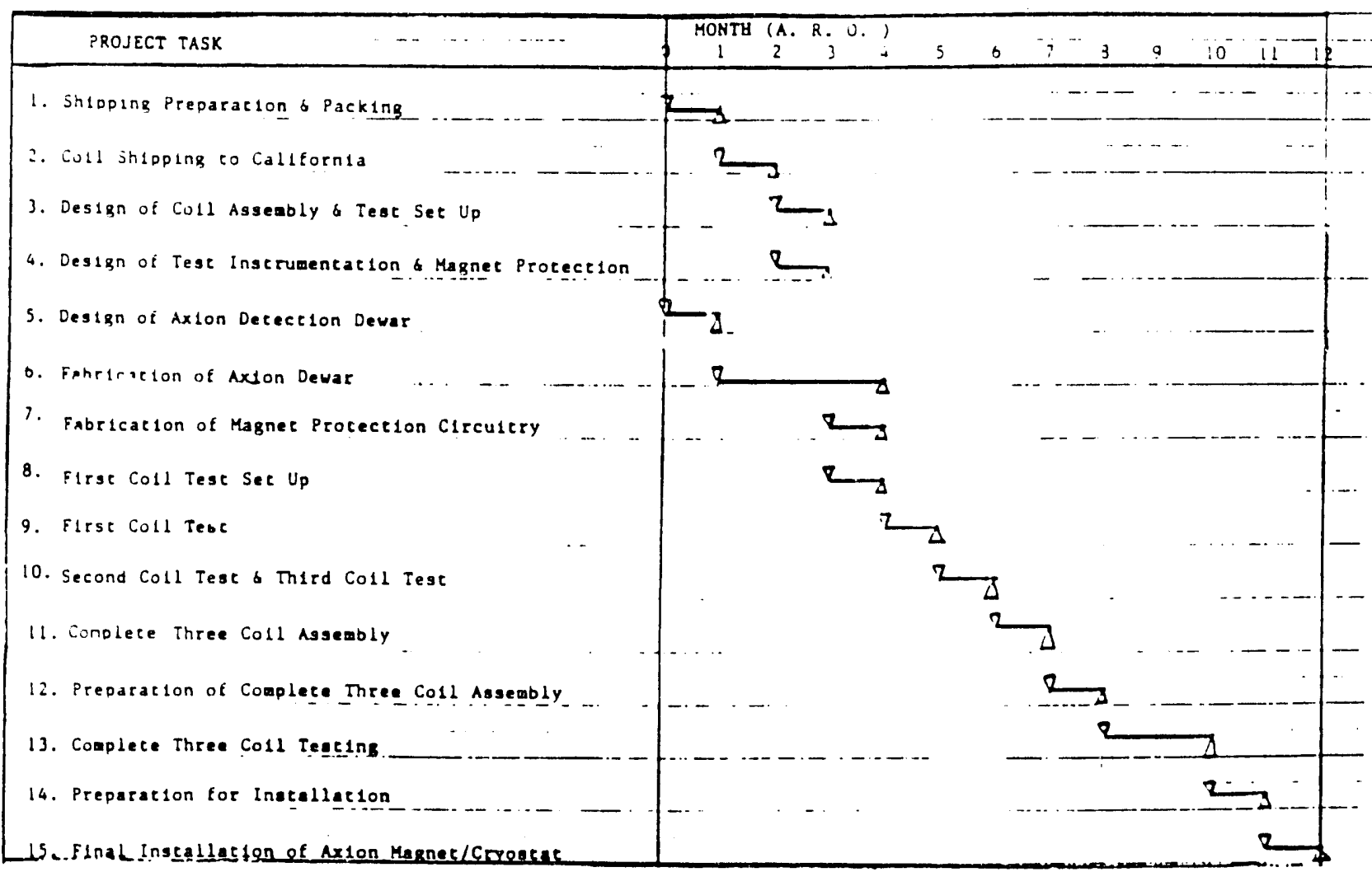

Figure 6. Timeline for the magnet and cryostat work in FY93. 


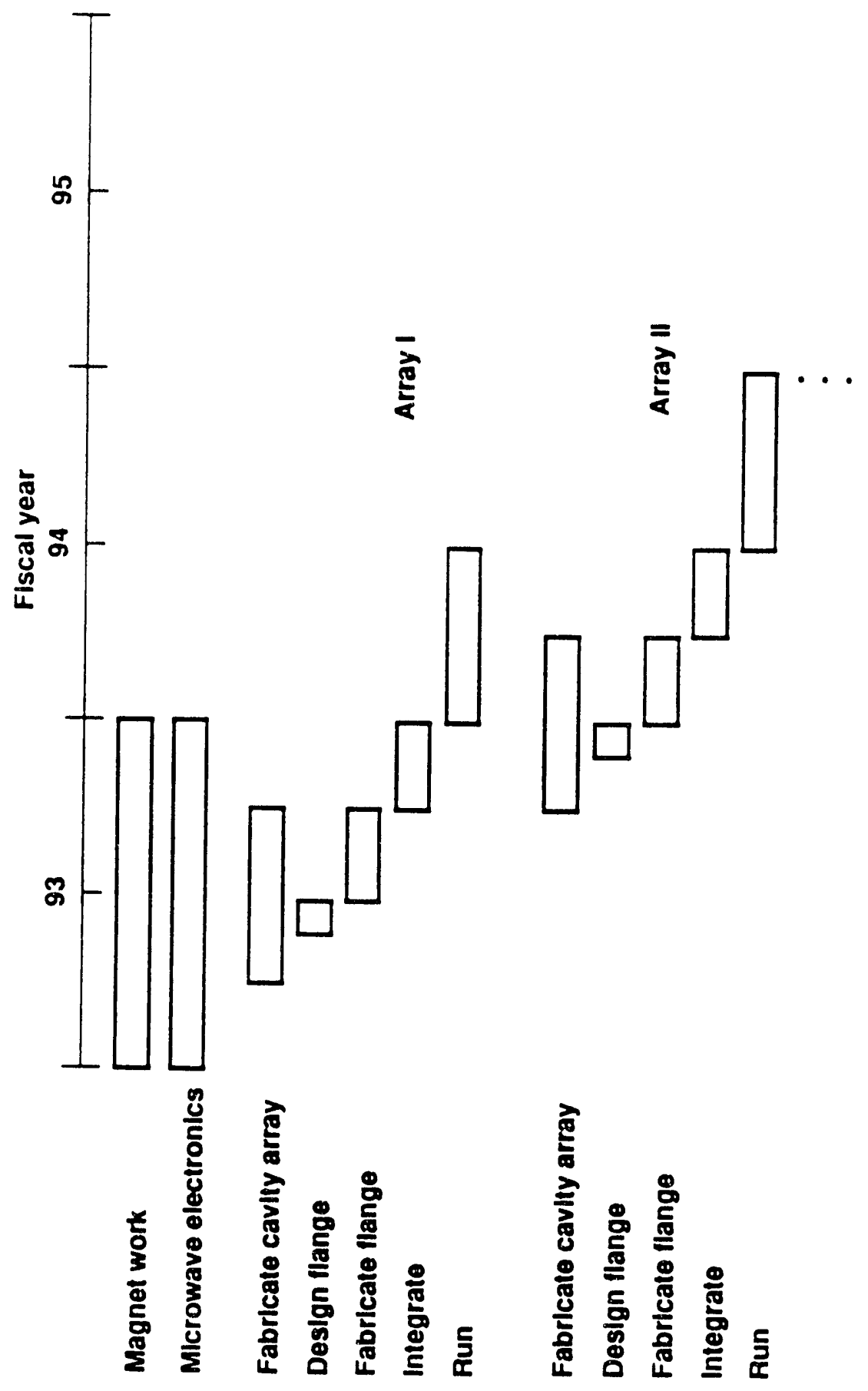

Figure 7. Timeline for construction and operation of the axion search experiment. 


\section{Reterences}

[1] "Preliminary Separation of Galactic and Cosmic Microwave Emission for the COBE-DMR", C.L. Bennett et al, submitted to Astrophysical Journal Letters, April, 1992

[2] "Structure in the COBE-DMR First Year Maps", G.F. Smoot et al, submitted to Astrophysical Journal Letters, April, 1992

[3] "Interpretation of the CMB Anisotropy Detected by the COBE DMR", E.L. Wright et al, submitted to Astrophysical Journal Letters, April, 1992

[4] R.D. Peccei and H. Quinn, Phys. Rev. Lett. 38 (1977) 1440; Phys. Rev. D16 (1977) 1791

[5] S. Weinberg, Phys. Rev. Lett. 40 (1977) 223

[6] F. Wilczek, Phys. Rev. Lett. 40 (1977) 279

[7] P. Sikivie, Phys. Rev. Lett. 51 (1983) 1415

[8] S. DePanfilis et al, Phys. Rev. Lett. 59 (1987) 839; Phys. Rev. D40 (1989) 3153

[9] C. Hagmann et al, Phys. Rev. D42 (1990) RC1297

[10] M.S. Turner, Phys. Rep. 197 (1990) 67

[11] G.G. Raffelt, Phys. Rep. 198 (1990) 1

[12] M. Dine, W. Fischler, M. Srednicki, Phys. Lett. 104B (1981) 199; A.P. Zhitnitskii, Sov. J. Nucl. Phys. 31 (1980) 260. This model is often abbreviated as 'DFSZ', as is in this proposal.

[13] J. Kim, Phys. Rev. Lett. 40 (1977) 223; M.A. Shifman, A.I. Vainshtein, V.I. Zakharov, Nucl. Phys. B166 (1980) 493. This model is often abbreviated at 'KSVZ', as is in this proposal.

[14] C. Hagmann, Thesis, University of Florida (1990)

[15] C. Hagmann et al, Rev. Sci. Instrum. 61 (1990) 1076

[16] J.E. Kim, private communication (1991)

[17] K. van Bibber et al, Proc. of the Workshop on Astroparticle Physics, Santa Monica, November 28-December 1, 1990, World Scientific (1992) 154, D. Cline, R. Peccei, ed; K. van Bibber et al, Particles and Fields '91, August 18-22,1991, Vancouver, B.C., World Scientific (1992) 992, D. Bryman, M. Comyn, ed. 


\section{KARLA VAN BIB BER}

April, 1992

Biographical

Date of Birth:

Citizenship:

$12 / 05 / 50$

D.O.E. Q-Clearance:

U.S.A.

03/86-

\section{Education}

9/68-6/72 B.S. Physics, B.S. Mathematics, MIT

9/72-9/76 PhD Physics, MIT

Present Occupation

10/85 Senior Physicist, E-Division/Physics, LLNL

7/91- Group Leader for High Energy Physics, E-Division/Physics

Previous Work Experience

1/80-9/85 Assistant Professor of Physics, Stanford University

6/77-12/79 Postdoctoral Researcher, Nucl. Sci. Div., LBL

9/77- 6/78 Lecturer, Dept. of Nucl. Eng., U.C. Berkeley

9/76- 6/77 Instructor, Dept. of Physics, MIT

Professional Societies

American Physical Society

Honors

3/82-9/85 Alfred P. Sloan Research Fellow

Service

1992-pres. Member, Physics Department LDRD Committee

1990-pres. Member, SLAC Users Organization Executive Committee

1986-1989 LLNL Lab-Wide Institutional and Research Committee, ViceChairman, FY89, Chairman, FY90

1983-1985 Chairman, Exec. Comm., LBL 88" Cyclotron Users Group

1983-1985 Phi Beta Kappa Executive Council, Stanford University, President, '84-' 85

1980-1982 APS Division of Nuclear Physics Program Committee 


\section{Research Interests}

High energy electron scattering from nucleons and nuclei.

Experimental possibilities for detection of axions or other light bosons.

Inelastic electron, hadron scattering to giant resonances and their decay.

\section{Relevant Publications}

1.) An Experiment to Produce and Detect Light Pseudoscalars, K. van Bibber, iv. Dagdeviren, S. Koonin, A.K. Kerman, H.N. Nelson Phys. Rev. Lett. 59, 759 (1987)

2.) A Practical Laboratory Detector for Solar Axions, K. van Bibber, P.M. McIntyre, D.E. Morris, G.G. Raffelt, Phys. Rev. D39, 2089, (1989), and Proceedings of the Workshop on Cosmic Axions, April 13-14, 1989, BNL, ed. C. Jones, A. Melissinos, p. 98 (World Scientific, 1990)

3.) A Proposed Search for Dark-Matter Axions in the 0.6-16 $\mu \mathrm{eV}$ Range, K. van Bibber, P. Sikivie, N.S. Sullivan, D.B. Tanner, M.S. Turner, D.M. Moltz, Proc. of the Workshop on Astroparticle Physics, Santa Monica, November 28December 1, 1990, World Scientific (1992) p. 154, D. Cline, R. Peccei ed.; also in Particles and Fields '91, August 18-22, Vancouver, B.C., World Scientific (1992) p. 992, D. Axen, D. Bryman and M. Comyn, ed. 
Curriculum Vitae for Wolfgang Stöffl

\section{PRESENT POSITION:}

Nuclear Chemistry Division

Lawrence Livermore National !.aboratory

L-396

Livermore, CA 94550

(510) $422-7312$

\section{PERSONAL:}

Citizenship: German

PROFESSIONAL MEMBERSHIPS:

American Physical Society

American Vacuum Society

EDUCATION:

9/76-3/80, Tech:ucal University Munich Ph.D

(Physics) awarded $3 / 80$

9/70-6/76, Technical University Munich Diploma.

(Physics) awarded 6/76

\section{PREVIOUS RESEARCH EXPERIENCE:}

Post-Doctoral Research Associate, Florida State University, Tallahassee, Florida $4 / 80-7 / 83$

\section{GENERAL RESEARCH INTERESTS:}

Neutrino Physics, Astrophysics, Nuclear Detector Development

\section{PUBLICATIONS AND OTHER PAPERS:}

Search for Lepton Flavour Violation in $Z^{0}$ decays.

L3 Collaboration, LEP, CERN Geneva.

(1991) Physics Letters B271 453

Quadrupole Moments of Low Energy Isomers in 210Po.

J.A.Becker, A.Berger, J.Blomqvist, D.J.Decman, R.Estep ,E.Henry, K.H.Maier, L.G.Mann, R.A.Meyer, N.A.F.M.Poppelier, N.Roy, W.Stöffl, G.L.Struble,L.D.Wood (1991) Nuclear Physics A522, 483

Atomic Electron Binding Energies of Multiply Charged Krypton Ions by Internal-ConversionElectron Spectrosco;y.

D. J. Decman and W. Stöffl

(1990), Phys. Rev Lett. 64, 2767.

High Resolution K-Auger Spectra of Krypton from Gaseous Kr-83m.

D. J. Decman and W. Stöff 
(1990), LCRL JC 103615 ABS.

Measurement of the Natural Line Shape of Krypton Conversion Electrons from Gaseous Kr-83m. D. J. Decman and W. Stöff

(1990), LCRL JC 106042 SUM.

Electromagnetic Decay of $10^{+}$States and Yrast Isomers in $208 \mathrm{~Pb}$.

N.Roy, K.H.Maier, A.Aprahamian, J.A.Becker, D.J.Decman, E.A.Henry, L.G.Mann,

R.A.Mever, W.Stöff, G.L.Struble

(1989) Physics Letters B221, 1, 6

Levels in ${ }^{112}$ Pd and ${ }^{102} \mathrm{Mo}$.

R.Estep, R.K.Sheline, D.J.Decman, E.A.Henry, L.G.Mann, R.A.Meyer, W.Stöffl, L.E.Ussery, J.Kantele.

(1989) Physical Review C39, 1, 76

Decay of the $21 / 2^{-}$Isomer in ${ }^{211} \mathrm{Bi}$ and the Structure of the $v G^{2} 9 / 2 \pi H 9 / 2$ Levels.

K.H.Henry, A.Aprahamian, J.A.Becker, D.J.Becker, D.J.Decman, E.A.Henry, R.G.Lanier,

L.G.MAnn, R.A.Mever, K.Sales, N.Roy, W.Stöff, G.L.Struble, J.Blomqvist.

(1989) Zeitschrift für Physik A332, 263

Levels of the four lowest two-particle configurations in $210 \mathrm{~Pb}$ studied by in-beam $\gamma$-ray and conversion-electron spectroscopy with the ${ }^{209} \mathrm{Bi}(t, 2 n)$ reaction.

L.G.Mann, K.H.Maier, A.Aprahamian, J.A.Becker, D.J.Decman, E.A.Henry, R.A.Meyer,

N.Roy, W.Stöffl, G.L.Struble.

(1988) Physical Review C38, 74

In-beam Spectroscopy Using the $(t, p)$ Reaction: Recent Results Near $A=100$.

E.A.Henry, R.J.Estep, R.A.Meyer, J.Kantele, D.J.Decman, L.G.Mann, R.K.Sheline, W.Stöffl, L.E.Ussery

(1987) Physical Review C35, 4, 1485.

Measurement of EO Transitions in Neutron-Rich Nuclei near $A=100$.

R.J.Estep, R.K.Sheline, D.J.Decman, E.A.Henry, L.G.Mann, R.A.Meyer, W.Stöffl, L.E.Ussery, J.Kantele

(1987) Physical Review C35, 4, 1485

The Level Structure of ${ }^{155} \mathrm{Gd}$ from $(n, \gamma),(d, p)$ and $(d, t)$ studies.

$\therefore$..H.Schmidt, W.Stöff, T.von Egidy, P.Hungerford, H.J.Scheerer, K.Schreckenbach,

H.G.Börner, D.D.Warner, R.E.Chrien, R.C.Greenwood, C.W.Reich.

(1986), J.Phys. G: Nucl. Phys. 12, 411

Particle-Hole Multiplets in ${ }^{146} \mathrm{Gd}$ from In-Beam Studies of Non-Yrast Levels.

S.W.Yates, R.Julin, P.Kleinheinz, B.Rubio, L.G.Mann, E.A.Henry, W.Stöffl, D.J.Decman, J.Blomqvist.

(1986) Zeitschrift für Physik A324, 417

Differential Cross Sections of ${ }^{3} \mathrm{H}(\mathrm{p}, \mathrm{n}){ }^{3} \mathrm{He}$ and of ${ }^{6} \mathrm{Li}(\mathrm{n}, \mathrm{t})^{4} \mathrm{He}$ by Using Triton Beams Between 5.95 and $19.15 \mathrm{MeV}$ and a Reevaluation of the P-T Neutron Production Cross Section up to 12 $\mathrm{MeV}$.

M.Drosg, G.Haouat, W.Stöff, D.M.Drake.

(1985) UCRL LA-10444-MS. 
Electromagnetic Transitions in $205 \mathrm{Hg}$.

K.H.Maier, W.Stöffl, R.K.Sheline, J.A.Becker, D.J.Decman, E.A.Henry, L.G.Mann,

G.L.Struble, L.E.Ussery, J.A.Cizewski, B.H.Erkkila.

(1985) Phusical Review C32, 4, 1416

Electron Spectroscopy in Coincidence with Protons from the $(t, p)$ Reaction.

D.J.Decman, E.A.Henry, J.Kantele, L.G.Mann, W.Stöffl, R.J.Estep, and L.E.Ussery

(1984),Nuclear Instruments and Methods, 219, 523.

Reinvestigation of the Gamma Branch from the U-238 Shape Isomer.

J. Kantele, W.Stöff, L.E.Ussery, D.J.Decman, E.A.Henry, R.J.Estep, R.W.Hoff, and L.C.Mann (1984) Physical Review, C29, 1693.

Measurement of the Quadrupole Moment of the $5^{-}$level in ${ }^{206} \mathrm{Hg}$.

K.H.Maier, M.Menningen, L.E.Ussery, T.W.Nail, R.K.Sheline, J.A.Becker, D.J.Decman,

R.G.Lanier, L.G.Mann, W.Stöff, G.L.Struble.

(1984) Physical Review C30, 5, 1702

$3291-\mathrm{keV} \mathrm{J} \pi=25 / 2^{+}$Level in $205 \mathrm{~T}$.

J.A.Becker, G.G.Lanier, L.G.Mann, G.L.Struble, K.H.Maier, L.E.Ussery, W.Stöff, T.W.Nail,

R.K.Sheline, I.A.Cizewski, B.H.Erkkila.

(1984) Physical Rview C29, 4, 1268

An Electron Spectrometer with a Superconducting Magnet Transporter for In-Beam Use.

W.Stöffl, E.A.Henry.

(1984) Nuclear Instruments and Methods, 227, 1, 77

Low-Lying States in $96 \mathrm{NB}$ from the $(t, \alpha)$ Reaction.

P.F.Cloessner, W.Stöff, R.K.Sheline, R.G.Lanier.

(1984) Physical Review C29, 2, 657

Observation of an EO Isomeric Transition from the U-238 Shape Isomer.

J. Kantele, W.Stöff, L.E.Ussery, D.J.Decman, E.A.Henry, R.W.Hoff, L.G.Mann, G.L.Struble

(1983) Physical Review Letters, 51, 91.

Electromagnetic properties of the isomer in ${ }^{210} \mathrm{~Pb}$.

D.J.Decman, J.A.Becker, J.B.Carlson, R.G.Lanier, L.G.Mann, G.L.Struble, K.H.Maier, W.Stöffl, R.K.Sheline.

(1983) Physical Review C28, 3, 1060

Structure of ${ }^{209} \mathrm{Bi}$ Deduced from the ${ }^{208} \mathrm{~Pb}(\mathrm{t}, 2 \mathrm{n} \gamma)$ Reaction.

K.H.Maier, T.Nail, R.K.Sheline, W.Stöffl, J.A.Becker, J.B.Carlson, R.G.Lanier, L.G.Mann, G.L.Struble, J.A.Cizewski, B.H.Erkkila.

(1983) Physical Review C27, 4, 1431.

In-Beam Gamma-Ray Studies with Tritons.

J.A.Becker, J.B.Carlson, R.G. Lanier, K.H.Maier, L.G.Mann, G.L.Struble, T.W.Nail,

R.K.Sheline, W.Stöffl. L.E.Ussery, J.A.Cizewski, B.Erkilla.

(1983) Nuclear Instruments and Methods 211, 379.

$2.102-\mathrm{MeV}$ Level in ${ }^{206} \mathrm{Hg}$ and the Spin Cyromagnetic Ratio of the 3-s Proton.

J.A.Becker, J.B.Carlson,, R.G.Lanier, L.G.Mann, G.L.Struble, K.H.Maier, L.Ussery, W.Stöffl,, T.Nail, R.K.Sheline, J.A.Cizewski. 
g Factor of the $\int^{\pi}=25 / 2^{+}$Isomer in ${ }^{205} \mathrm{Tl}$ and the Anomalous Orbital Magnetism of the Proton. K.H.Maier, J.A.Becker, J.B.Carlson, R.G.Lanier, L.G.Mann, G.L.Struble, T.Nail, R.K.Sheline, W.Stöffl, L.Ussery.

(1982) Physical Review Letters 48, 7, 466

Rotational-Vibrational Band Structure in ${ }^{155} \mathrm{Sm}$.

K.Schreckenbach, A.I.Namenson, W.F.Davidson, T.von Egidy, H.G.Börner, J.A.Pinston, R.K.Smither, D.D.Warner, R.F.Casten, M.L.Stelts, D.H.White, W.Stöffl.

(1982) Nuclear Physics A376, 149

Evidence for a Strongly Deformed Prolate Shape at $N=97$ from ${ }^{154} \mathrm{Eu}$ and ${ }^{152} \mathrm{Eu}(\mathrm{p}, \mathrm{t})$ Reactions R.G.Lanier, G.L.Struble, L.G.Mann,W.Stöffl, I.C.Oelrich, J.Scheerer, I.D.Proctor,

D.W.Heikkinen, R.H.Howell.

(1981) Physics Letters 99B, 123

Nuclear Levels in ${ }^{152}$ Eu.

T.von Egidy, W.Kaiser. W.Mampe, C.Hillenbrand, W.Stöff, R.C.Lanier, K.Mühlbauer, O.W.B.Schult, H.R.Koch, H.A.Baader, R.L.Mlekodaj, R.K.Sheline, E.B.Shera, J.Ungrin, P.T.Prokofiev, L.I.Simonova, M.K.Balodis, H.Seyfarth, B.Kardon, W.Delang, P.Göttel, D.Breitig, W.R.Kane, R.F.Casten, H.J.Scheerer, P.Glässl, E.Hünges, M.Löffler, H.Rösler, H.K.Vonach

(1978) Zeitschrift für Physik A 286, 341

Levels in ${ }^{154}$ Eu Populated by Thermal Neutron Capture. iN.Stöff, D.Rabenstein, K.Schreckenbach, T.von Egidy (1977) Zeitschrift für Physik A282, 97

Physics Colloquia at:

(1985) California Institute of Tecinnology (Pasadena)

(1989) University of Munich (Munich)

(1990) Institute Laue-Langevin (Grenoble)

(1990) Lawrence Berkeley Lab (Berkeley)

(1991) Massachusetts Institute of Technology (Cambridge)

(1992) Princeton University (Princeton)

(1992) Notre Dame University (South Bend)

(1992) U.C. Berkeley (Berkeley)

(1992) University of Washington (Seattle)

(1992) Invited Talk at the American Physical Society Spring Meeting in Washington D.C..

(1992) SLAC (Stanford) 
Block 1 - General Information

Proposal Title

Search for Pseudoscalar Cold Dark Matter

Proposal Type DE
Technical Area
Particle and Nuclear Physics

Proposal continued from FY92

Proposal tracking Ne

Block 2 - Principal Investigator

\begin{tabular}{|c|c|c|c|}
\hline \multirow{2}{*}{\multicolumn{2}{|c|}{$\begin{array}{r}\text { First/Last Name } \\
\text { Directorate }\end{array}$}} & Kari & van Bibber \\
\hline & & \multicolumn{2}{|l|}{ Physics } \\
\hline & Dept & \multicolumn{2}{|l|}{ Physics } \\
\hline & Div & \multicolumn{2}{|l|}{ E Division } \\
\hline
\end{tabular}

\begin{tabular}{rl|l|} 
L.Code & L-288 \\
Phone & $3-8086$ & \\
Emall & vanbibber1@llni.gov \\
Fax & $3-8086$ &
\end{tabular}

Block 3 - Responsible AD

Block 4 - Responsible Resource Mgr

Directorate

Physics
First/Last Name Marshall Mugge

L.Code L-403

Phone $3-1765$

_ Block 5 - Co-Investlgators

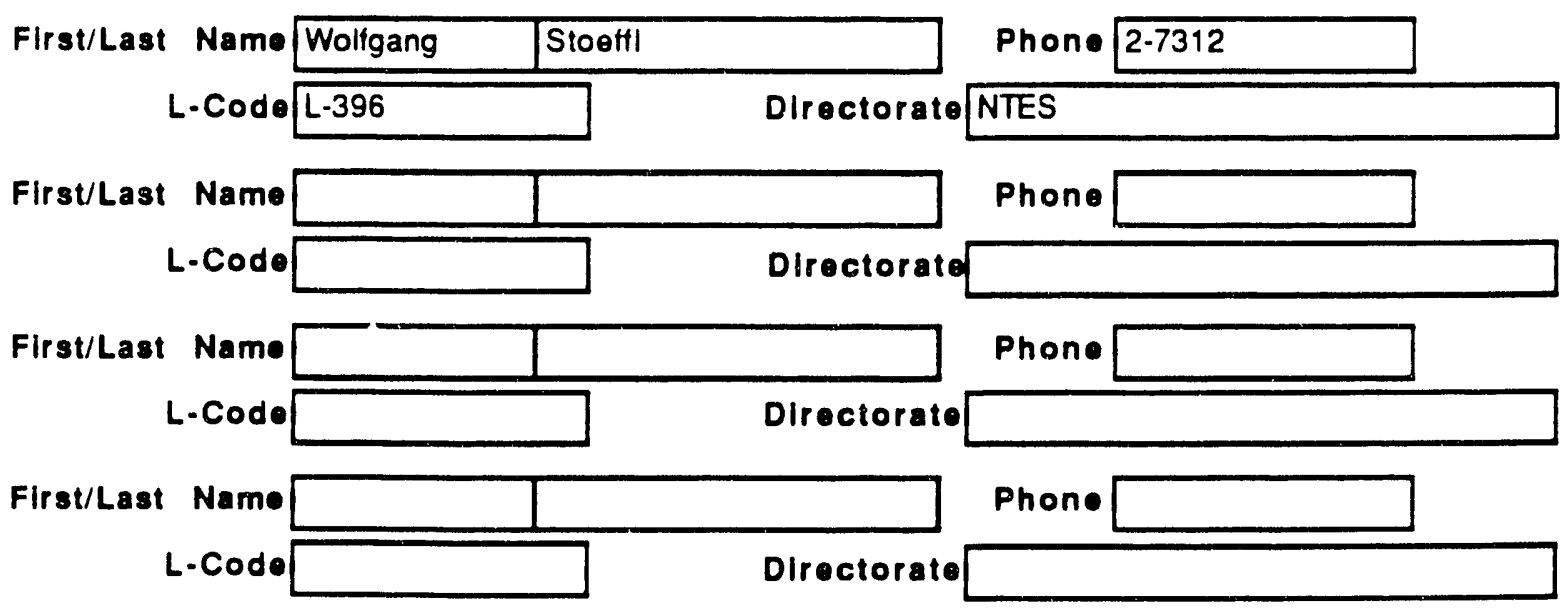


Block 6a - Project Description

Project Abstract

It is virtually certain that at least $90 \%$ of the mass of the universe is in a non-luminous form. Both dynamical evidence, and observation of primordial fluctuations in the cosmic microwave background support the hypothesis of an exactly closed universe, predominantly composed of 'cold dark matter'. One of the most attractive cold dark matter candidates is a previously undiscovered elementary particle called the axion. Dark-matter axions may be detected by their stimulated conversion to microwave photons in a tunable high- $Q$ cavity in a strong magnetic field. We propose to perform the first axion search that could possibly detect axions comprising our own galactic halo. This proposal captalizes on the availability of a compact high field superconducting magnet, a commitment from the INR/Russian Academy of Sciences for all the microwave cavities required for the experiment, and substantial university capital monies for the microwave electronics. The experiment would take data within one year, and run for two years thereafter. 
Block 6b - Project Description

Major Accomplishmonts and Results - FY 1992

$\checkmark$

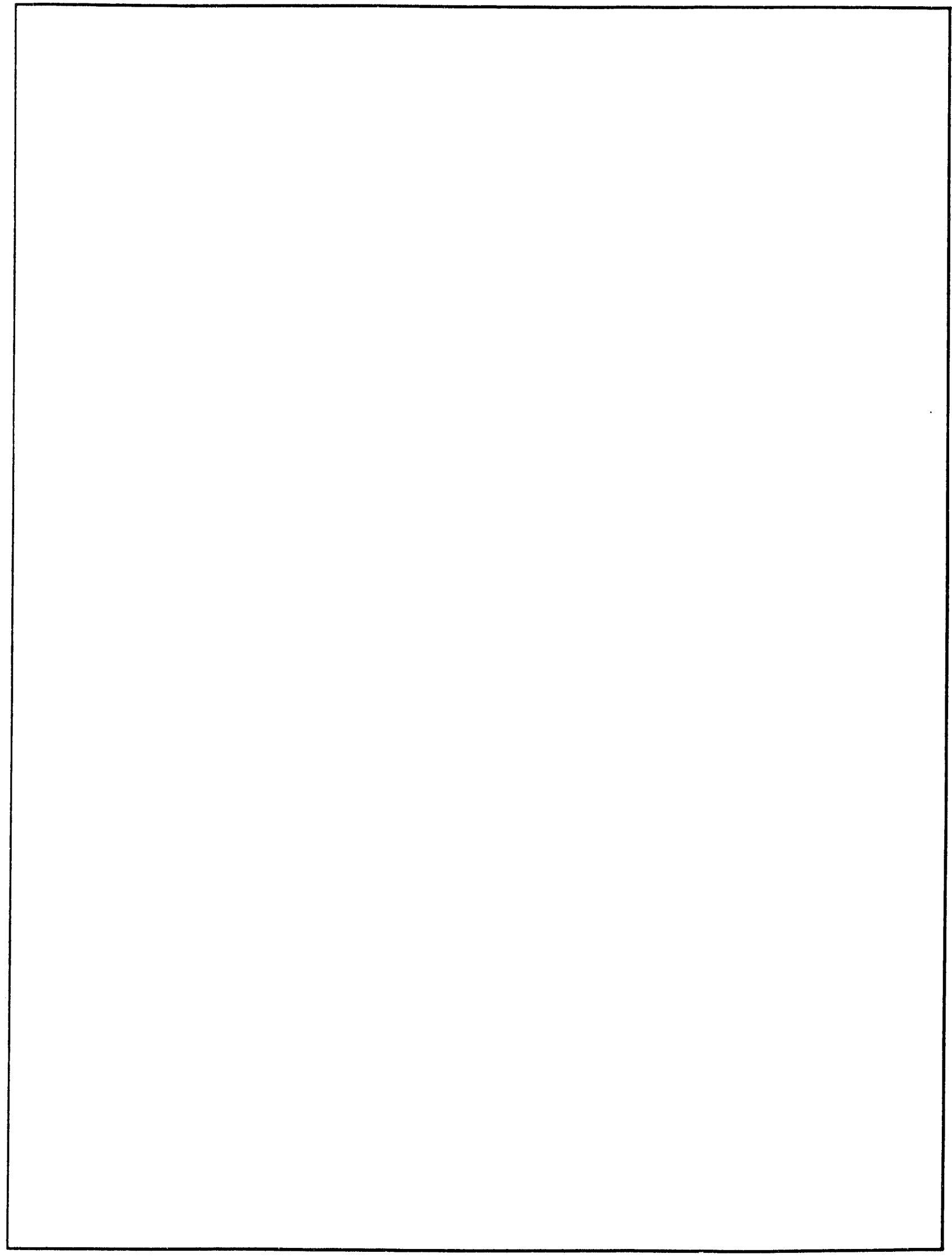


Block 6c - Project Description

Proposed Work and Anticipated/Desired Results - FY 1993

The first year will consist entirely of the construction and integration of the cavity microwave axion experiment. Early in the first year, we will let the contract for the construction of the cryostat, the mounting and testing of the NASA SUMMA magnets within it, and the refurbishing of the power supplies, control system and quench protection circuit. This work will be substantially complete by the end of FY 1993. We will design and fabricate the top flange for the cryostat from which the first cavity array will be suspended in the magnet. This will be complete in time for the delivery of the first cavity array by our INR collaborators. The microwave electronics will be procured, assembled and tested at MIT, and shipped to the laboratory. We will also conduct the search and appoint a postdoctoral researcher at LLNL in support of the project for the following two years. The experimental run with the first cavity array will begin early in the second year. 


\section{Get information for Blocks 7.13 from your resource manager}

Items wrth a bold outline will be automatically calculated by the system.

Block 7 - Manpower Expenses

\begin{tabular}{rrrr|}
\hline FY1993 & FY1994 & FY1995 \\
\hline 0.50 & 1.25 & 1.50 \\
\hline$\$ 44,772$ & $\$ 119,481$ & $\$ 155,571$ \\
\hline$\$ 3,855$ & $\$ 7,890$ & $\$ 8,276$ \\
\hline$\$ 33,579$ & $\$ 89,611$ & $\$ 116,678$ \\
\hline$\$ 82,206$ & $\$ 216,982$ & $\$ 280,525$ \\
\hline
\end{tabular}

E. Labor - Consultants

F. Labor - LBL

G. Labor - Post Doctorates.

H. Labor - Summer Hires

I. Labor - Supplemental.

J. Labor - Supplemental $+60.0 \%$ Overhead.

Other Labor Tota

\begin{tabular}{|l|r|r|}
\hline & & \\
\hline & $\$ 70,000$ & $\$ 73,430$ \\
\hline
\end{tabular}

Block 8 - Capital Equipment Summary Requests Over $\$ 5000$ (FY1993-FY1995)

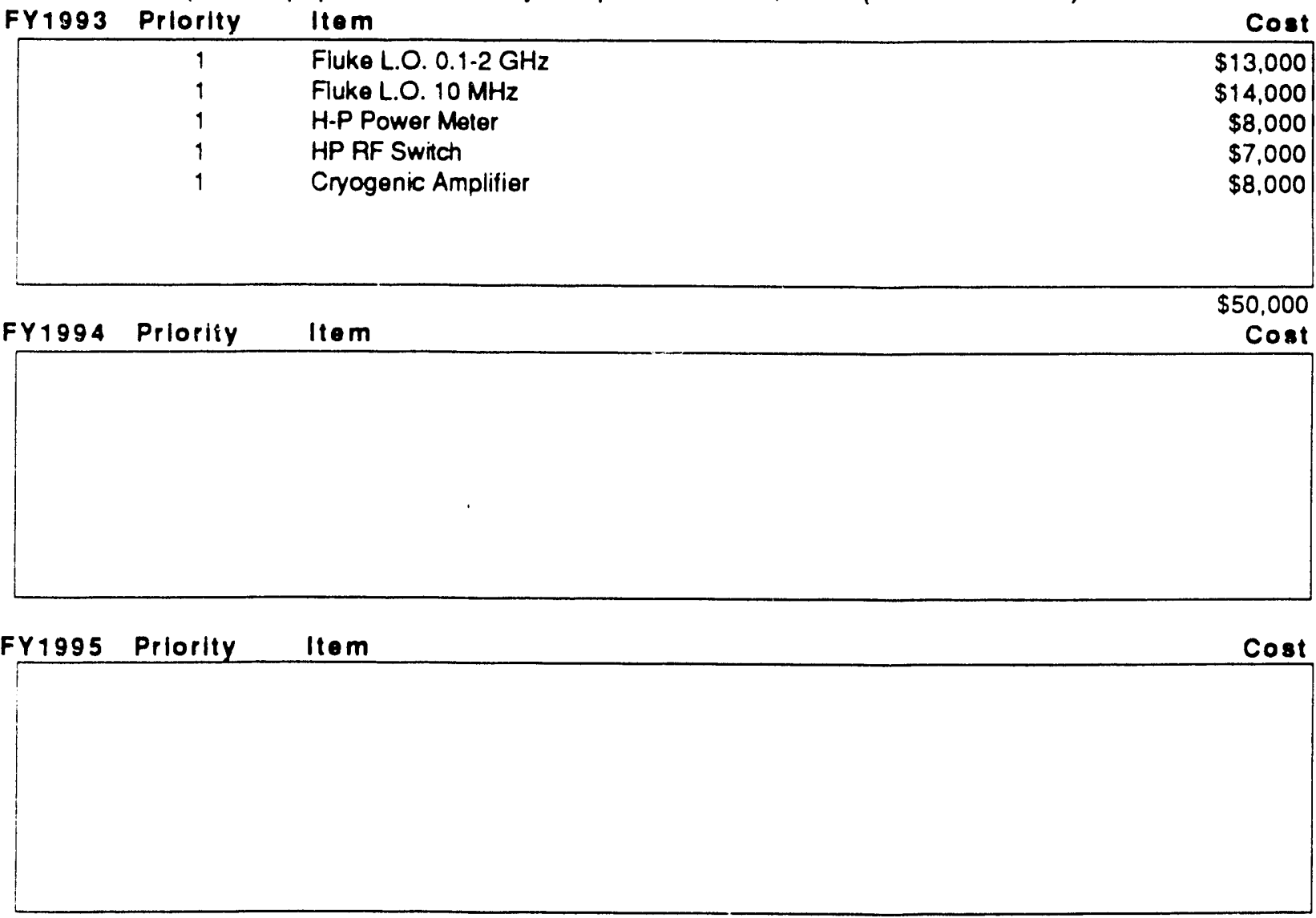



A. Procurements
FY1993
FY1994
FY1995
$\$ 205,000$
$\$ 210,000$
$\$ 10,000$

Block 10 - Expenses

A. Foreign Travel

B. Domestic Travel.

C. Siores.

D. Other

Total

Expenses.

\begin{tabular}{|c|c|c|c|}
\hline & FY1993 & FY1994 & FY1995 \\
\hline & $\$ 3,000$ & $\$ 12,600$ & $\$ 19,800$ \\
\hline & $\$ 1,000$ & $\$ 2,000$ & $\$ 2,000$ \\
\hline & $\$ 1,000$ & $\$ 5.000$ & $\$ 3,000$ \\
\hline & & $\$ 50,000$ & $\$ 55,000$ \\
\hline 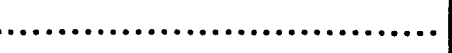 & $\$ 5,000$ & $\$ 69,600$ & $\$ 79,800$ \\
\hline
\end{tabular}

Block 11 - Services Facilitles

\begin{tabular}{|c|c|c|c|}
\hline & FY1993 & FY1994 & FY1995 \\
\hline A. Materials Fabrication Division (MFD)................. & \$o & $\$ 0:$ & \$o \\
\hline B. Technical Information Department (TID)............. & $\$ 1.000$ & $\$ 1,000$ & $\$ 2.000$ \\
\hline 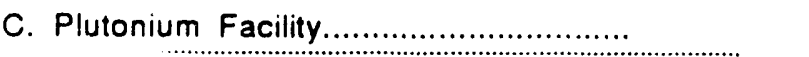 & So & $\$ 0$ & \$o \\
\hline D.Other....... & $\$ 0$ & $\$ 0$ & so \\
\hline Total Services/Faclllty......................... & $\$ 1,000$ & $\$ 1,000$ & $\$ 2,000$ \\
\hline
\end{tabular}

Block 12 - Computers

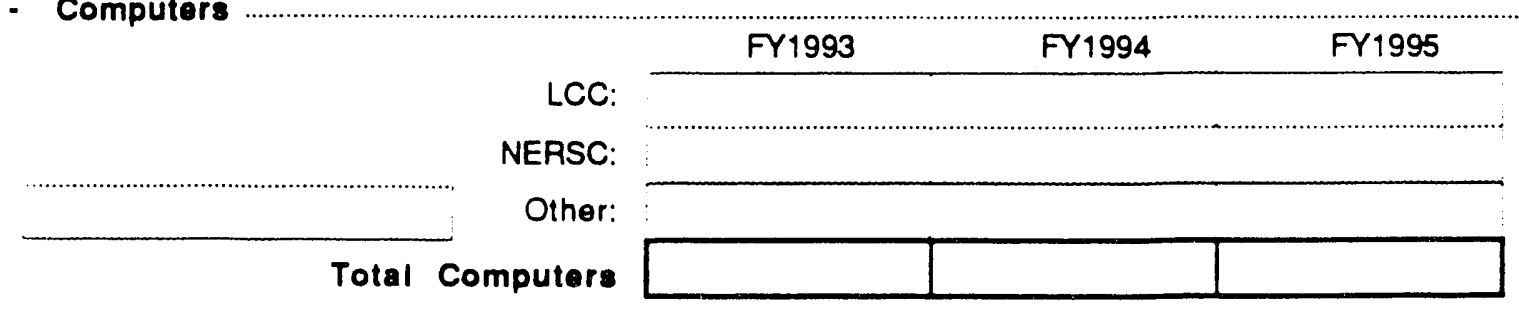




\begin{tabular}{|c|c|c|c|}
\hline & FY1993 & FY1994 & FY1995 \\
\hline $\begin{array}{l}\text { A. Project Operational } \\
\quad \text { (Blocks 7,9.10,11, and } 12 \ldots \ldots \ldots \ldots \ldots \ldots\end{array}$ & $\$ 293,206$ & $\$ 567.582$ & $\$ 445.755$ \\
\hline $\begin{array}{l}\text { B. Laboratory General Expense } \\
\text { (3\% of the values in A. for } 3 \text { years)........... }\end{array}$ & $\$ 8,796$ & $\$ 17,027$ & $\$ 13,373$ \\
\hline 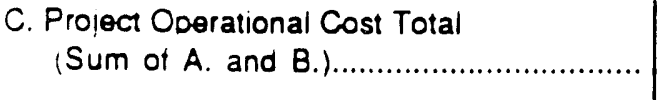 & $\$ 302,002$ & $\$ 584,609$ & $\$ 459,128$ \\
\hline 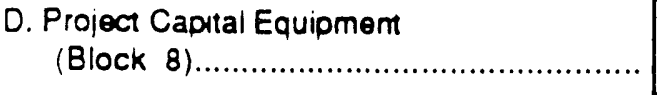 & $\$ 50,000$ & & \\
\hline Project Grand Total $(C+D) \ldots \ldots \ldots . .$. & $\$ 352,002$ & $\$ 584,608$ & $\$ 459,128$ \\
\hline
\end{tabular}



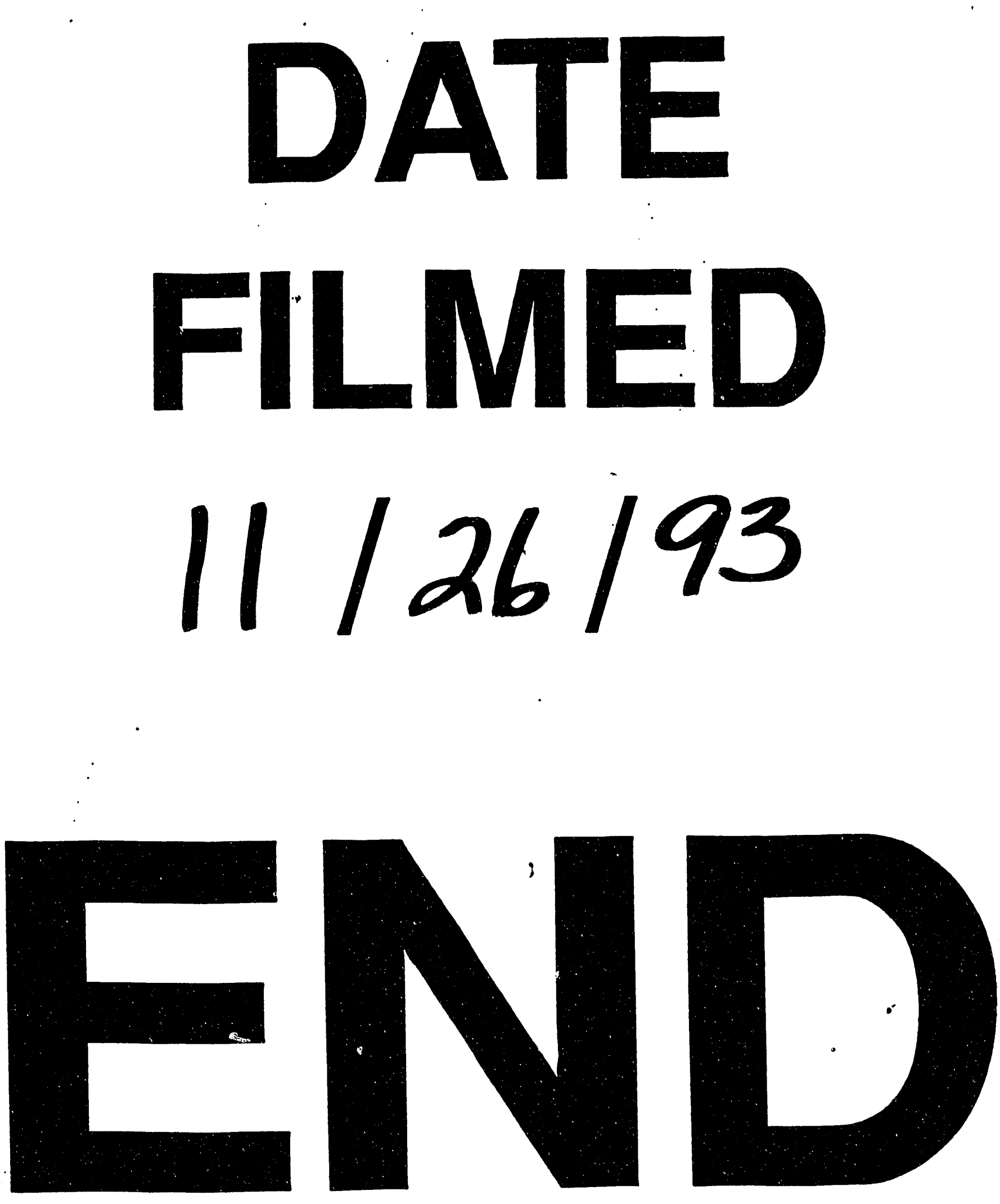


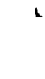

